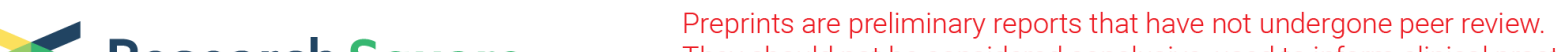 Research Square
or referenced by the media as validated information.
}

\section{Revealing the host antiviral protein ZAP-S as an inhibitor of SARS-CoV-2 programmed ribosomal frameshifting}

Matthias Zimmer

Helmholtz Institute for RNA-based Infection Research

Anuja Kibe

Helmholtz Institute for RNA-based Infection Research

Ulfert Rand

Helmholtz Centre for Infection Research https://orcid.org/0000-0002-5946-4921

\section{Lukas Pekarek}

Helmholtz Institute for RNA-based Infection Research

\section{Luka Cicin-Sain}

Helmholtz Centre for Infection Research https://orcid.org/0000-0003-3978-778X

Neva Caliskan ( $\sim$ Neva.Caliskan@helmholtz-hiri.de)

Helmholtz Institute for RNA-based Infection Research https://orcid.org/0000-0003-0435-4757

\section{Article}

Keywords: SARS-CoV-2, programmed ribosomal frameshifting (PRF), RNA-based gene regulation.

Posted Date: June 1st, 2021

DOI: https://doi.org/10.21203/rs.3.rs-563757/v1

License: (1) This work is licensed under a Creative Commons Attribution 4.0 International License.

Read Full License

Version of Record: A version of this preprint was published at Nature Communications on December 10th, 2021. See the published version at https://doi.org/10.1038/s41467-021-27431-0. 


\section{Abstract}

Programmed ribosomal frameshifting (PRF) is a fundamental gene expression event in many viruses including SARS-CoV-2, which allows production of essential structural and replicative enzymes from an alternative reading frame. Despite the importance of PRF for the viral life cycle, it is still largely unknown how and to what extent cellular factors alter mechanical properties of frameshifting RNA molecules and thereby impact virulence. This prompted us to comprehensively dissect the interplay between the host proteome and the SARS-CoV-2 frameshift element. Here, we reveal that zinc-finger antiviral protein (ZAP$S$ ) is a direct and specific regulator of PRF in SARS-CoV-2 infected cells. ZAP-S overexpression strongly impairs frameshifting and viral replication. Using in vitro ensemble and single-molecule techniques, we further demonstrate that ZAP-S directly interacts with the SARS-CoV-2 RNA and ribosomes and interferes with the folding of the frameshift RNA. Together these data illuminate ZAP-S as de novo host-encoded specific inhibitor of SARS-CoV-2 frameshifting and expand our understanding of RNA-based gene regulation.

\section{Introduction}

The novel severe acute respiratory syndrome-related coronavirus (SARS-CoV-2), the causal agent of Coronavirus Disease 2019 (COVID-19), emerged rapidly to become a global threat to human health ${ }^{1}$. Global analyses of RNA- and protein-interaction networks have increased our understanding of SARSCoV-2 viral replication in a short time ${ }^{2,3}$. However, there is a lack of detailed mechanistic understanding of the interplay between RNA-protein complexes, which could inform the design of novel antivirals. Here, functionally important RNA elements of the viral genome represent ideal targets due to their evolutionary conservation. One of those well-conserved RNA elements is the programmed ribosomal frameshift site.

A hallmark of infections by the SARS-CoV-2 and many other viruses is the -1 programmed ribosomal frameshifting (-1PRF) event which allows translation of multiple proteins from the same transcript. Frameshifting increases the coding potential of the genomes and is often used to expand the variability of proteomes, adapt to changing environments, or ensure a defined stoichiometry of protein products 4,5 . In coronaviruses, -1 frameshifting on the $1 a / 1 b$ gene is fundamental for efficient viral replication and transcription of the viral genome. In cells, efficiency of this frameshifting event varies between $20-40 \%$ 6,7 . Programmed ribosomal frameshifting relies on the presence of a slippery heptameric sequence (in coronaviruses U UUA AAC) and an RNA secondary structure such as a pseudoknot (Fig. 1A). Mutations in the slippery sequence and downstream RNA structure drastically impair frameshifting efficiency ${ }^{8,9}$.

Traditionally, efforts to understand the mechanism of - 1PRF focused on cis-acting modulatory elements. Previous work in purified translation systems explained in unprecedented detail how ribosome pausing on the slippery codons may lead to a kinetic partitioning and favor movement of translating ribosomes to an alternative reading frame ${ }^{6,10}$. It has been shown that - 1PRF may occur during a late stage of the tRNA translocation step with the stimulatory element causing ribosomes to become trapped in an unusual conformation that is relieved by either the spontaneous unfolding of the blockade or a -1 
slip on the mRNA ${ }^{6,10}$. Recently, it is becoming clear that cis-acting elements are not the only determinants of frameshifting in cells and trans-acting viral and cellular factors as well as small molecules or oligonucleotides can alter frameshifting levels ${ }^{11-13}$. Despite this momentum, fundamental questions such as how pertinent RNA-binding factors are for frameshifting processes in general and how exactly these interactions alter the mechanical properties of RNA as well as the choice of the reading frame remain to be exploited.

Based on current knowledge, there would be at least three potential routes to modulate frameshifting by trans-acting factors. First, the binding of the factor can transform the downstream RNA element to a more stable roadblock, which was shown for cardiovirus $2 \mathrm{~A}$, poly-(C) binding protein and some small molecules such as the NCT-8 $11,12,14$. In these cases, the specific interaction of the factor with the nucleotides downstream of the slippery codons leads to an increase in frameshifting. Alternatively, eukaryotic release factors such as eRF1 alone or eRF1/3 recruited by Shiftless (SFL) to the HIV-1 frameshift site were shown to target stalled ribosomes ${ }^{15,16}$. In this case, different from the first group of regulators the interaction of both SFL and release factors was not dependent on the identity of the frameshift RNA. Therefore, it remains to be solved how the frameshifting ribosome complexes would be recognized by these trans-acting factors. A third route could potentially work through remodeling or destabilization of the frameshifting RNA elements through direct interactions between the RNA and the trans-factor. However, so far there has been no cellular or viral factor reported to affect frameshifting efficiency (FE) through this route.

These prompted us to comprehensively identify and study direct interactions between the host cell proteome and the SARS-CoV-2 frameshifting RNA element. Firstly, to decipher interactors of the frameshifting RNA element, we employed an in vitro RNA-antisense capture and mass spectrometrybased screen ${ }^{17}$. Through this approach, we identified the short isoform of zinc-finger antiviral protein (ZAP-S, ZC3HAV1), as a prominent RNA interaction partner. We demonstrated that ZAP-S acts as a hostencoded inhibitor of SARS-CoV-2 1a/1b frameshifting in vivo and in vitro. Intriguingly, ZAP-S overexpression reduced the replication of SARS-CoV-2 by more than $90 \%$, highlighting the importance of the protein in the viral life cycle. The effect of ZAP-S on SARS-CoV-2 frameshifting was specific, because barring the closely related SARS-CoV-1, other viral and cellular PRF levels were not affected by ZAP-S in vivo. Using a multidisciplinary approach, we further probed this effect and revealed important clues on molecular principles of frameshifting downregulation by ZAP-S. Amongst them, we show that ZAP-S can alter the physical properties of the PRF RNA, which brings a unique dimension to frameshift mechanisms. Our study highlights for the first time that the expression of the SARS coronavirus ORF1a/1b, can be directly and specifically modulated by a host-encoded RNA-binding protein during infection. These findings provide substantial new insights on PRF regulation and the interplay between SARS-CoV-2 replication and host defense, thereby paving the way for novel RNA-based therapeutic intervention strategies.

\section{Results}




\section{SARS-CoV-2 PRF RNA capture identifies novel host interactors}

To identify potential cellular RNA-binding proteins (RBPs) that interact with the - 1PRF element of SARSCoV-2, an in vitro synthesized RNA fragment corresponding to nucleotides 13456-13570 of the SARSCoV-2 genome was incubated with lysates of SARS-CoV-2-infected and uninfected Calu-3 cells and naïve HEK293 cells (Fig. 1B) ${ }^{17}$. Calu-3 cells are lung epithelial cells that are commonly used to study CoV infection ${ }^{18}$. HEK293 cells are routinely used to study RNA-protein interactomes, therefore they represented an ideal system to assess possible cell-based variations ${ }^{19}$. To exclude any non-specific binders, we used an 80 nucleotides long non-structured RNA as a control. RNAs were captured by a biotinylated antisense DNA-oligo, and interacting proteins were identified by LC-MS/MS (liquid chromatography tandem mass spectrometry) analysis (Fig. 1B, C).

In our SARS-CoV-2 frameshift RNA capture, more than 100 proteins were at least two-fold enriched. According to our $\mathrm{GO}$ term analysis, the majority $(80 \%)$ of identified hits have been described as RNAbinding proteins (Supplementary Fig. 1A). As for viral proteins, we saw an enrichment of the viral nucleocapsid protein $(\mathrm{N})$ in infected lysates, which is a well-described RNA-binding protein ${ }^{20}$. In addition, $35 \%$ and $30 \%$ of the enriched RBPs were involved in splicing and ribosome biogenesis, respectively (Supplementary Fig. 1A). Among those, 19 proteins were common to infected and uninfected Calu-3 cells, 18 hits were identified only in HEK293 cells, 15 were captured only in uninfected Calu-3 cells, and 40 were present only in infected Calu-3 cells (Supplementary Fig. 1B). The core interactome of 9 proteins identified in all three cell systems encompasses well-described post-transcriptional regulators (Fig. 1C, D, Supplementary Fig. 1C, Supplementary Table 1). Proteins recently identified in genome-wide interactome studies as direct RNA interaction partners for SARS-CoV-2 were selected for downstream functional characterization ${ }^{3,20-22}$. Several of these have been shown to play a role in RNA processing, including splicing (such as HNRNPs $\mathrm{F}, \mathrm{H} 1$, and H2), RNA trimming (POP1) and RNA decay (ZAP) ${ }^{23-25}$. It is well known that some nuclear RBPs can localize and may carry out diverse functions to regulate gene expression in the cytoplasm ${ }^{23}$. For instance, HNRNP A2/B1 binds to VEGF-A mRNA and thereby affects recoding through readthrough ${ }^{26}$, and HNRNPD is involved in RNA folding and replication of Flaviviruses 27. Translational regulators included IGF2BP1, ELAVL1, DHX36, and SSB ${ }^{28,29}$. ELAVL1 is a cofactor which ensures translational fidelity in the context of uORFs ${ }^{30}$. DHX36 functions as a multifunctional helicase and is involved in translation and innate immunity ${ }^{31,32}$. ZAP is an antiviral protein with two isoforms (ZAP-S and ZAP-L), both of which are implied in various RNA-related mechanisms, including RNA decay and translation ${ }^{25,33}$. In addition to the aforementioned proteins, three more hits were selected for further analysis based on their fold enrichment in infected Calu-3 lysates. These included the RNA-binding protein GRSF1, the poly(A) polymerase PAPD4 as well as GNL2 which has been implied in ribosome biogenesis ${ }^{34-36}$. Unlike IGF2BP1, the closely related IGF2BP3 was enriched to a much lesser extent $\left(\log _{2}\right.$ enrichment 0.4-0.7) and, hence, was included as a control for the functional assays.

\section{RNA interactors specifically inhibit SARS-CoV-2 frameshifting in cells}


In order to explore the potential role of the RNA binders in SARS-CoV-2 frameshifting, we designed an in vivo fluorescence-based - 1PRF assay. In this assay, the expression of the first ORF EGFP in the 0-frame would be constitutive, whereas the expression of the following ORF mCherry would depend on - 1PRF occurring at the preceding SARS-CoV-2 1a/1b frameshifting fragment (Fig. 2A). As controls, we used a construct lacking the - 1PRF stimulatory sequence, and the mCherry gene is placed either in - 1 or inframe with respect to EGFP (Fig. 2A, B). Frameshift efficiencies were calculated as the ratio of mCherry to EGFP in the test construct normalized to the in-frame control (see also Materials and Methods). To study the effect of the trans-acting factors on SARS-CoV-2 1a/1b frameshifting, cells were co-transfected with both the dual-fluorescence reporter plasmid and the plasmid encoding the putative trans-factor as an $\mathrm{N}$ terminal ECFP fusion. This was advantageous over luciferase-based assays, because fluorescence readout allowed gating for the ECFP + cells, which express the protein of interest. To benchmark the assay, a vector expressing only ECFP was used as a control to compensate for the spectral overlap between ECFP and EGFP. In addition, vector expressing ECFP-SFL, a previously described inhibitor of 1PRF in SARS-CoV-2, was used as a positive control ${ }^{3}$. Using this fluorescence reporter system, the frameshifting efficiency (FE) of SARS-CoV-2 was measured to be ca. 35\% in HEK293 (Fig. 2C, D,

Supplementary Fig. 2A, B, Supplementary Table 2), which agreed well with the published FE for SARSCoV-1 as well as the SARS-CoV-2 $7,9,37$. As expected, deletion of the nucleotides corresponding to the predicted stem 2 of the pseudoknot as well as deletion of the region corresponding to the predicted stem 2 and 3 led to the abrogation of - 1PRF, in line with minimal sequence requirements for frameshifting of other coronaviruses (Supplementary Fig. 2A) ${ }^{9}$.

Among the candidate PRF regulators examined, we observed no change in FE with GNL2, HNRNPF, IGF2BP1 or SSB which points to the fact that binding to the stimulatory RNA element is not sufficient for modulating PRF. Also, several proteins that were not significantly enriched in the interactome capture were not able to lead to significant changes in FE corroborating the specificity of the flow-cytometrybased frameshifting assay (Supplementary Fig. 2B). On the other hand, several candidates such as SFL, HNRNPH1, HNRNPH2, ZAP-L and ZAP-S, showed a substantial reduction in FE by up to $50 \%$ (Fig. 2C). Other candidates like GRSF1 and PAPD4 led to a slight but not statistically significant increase in FE. The candidates with the strongest effect on FE were HNRNPH1, HNRNPH2 and ZAP-S. In order to test whether these RBPs were functionally relevant, we measured their relative expression levels using quantitative RTPCR in SARS-CoV-2 uninfected versus infected Calu-3 cells at 72 hours post-infection. Only ZAP showed a significant ca. 20-fold increase of mRNA levels upon infection (Fig. 2D).

ZAP is a well-known multi-functional antiviral protein with an established role in the innate immune response against alphaviruses, filoviruses, HIV-1, hepatitis $B$ virus and influenza virus ${ }^{38}$. Whilst the mechanism of action for ZAP seems to revolve around the modulation of RNA stability ${ }^{39-41}$, a more recent study implies its role in the maturation of viral particles ${ }^{42}$. The ZAP gene produces two isoforms, ZAP-S and ZAP-L, differing only in their N-terminal domains. The two ZAP isoforms were suggested to carry out different functions in infections ${ }^{33}$. While the longer, constitutively expressed isoform ZAP-L preferentially targets viral RNAs, the shorter isoform ZAP-S has been identified as an immune-regulatory 
protein that targets interferon mRNAs ${ }^{33}$. In our assay, both isoforms were tested simultaneously, and we observed the decrease in frameshifting upon overexpression of the ZAP-S and ZAP-L. In both cases, the level of 0-frame product EGFP was not changed (Supplementary Table 2). However, the - 1PRF-inhibitory effect of ZAP-S was significantly stronger than the one by ZAP-L (Fig. 2C). Therefore, we decided to follow up the interactions of ZAP-S as a frameshift regulator in detail.

First, to further corroborate the specificity of ZAP-S for the SARS-CoV-2 frameshift element, we tested whether the overexpression of ZAP-S affects - 1PRF of other mRNAs, e.g., different Betacoronaviruses (SARS-CoV-1, MERS-CoV, Bat-CoV-273), Arboviruses (West Nile Virus (WNV), Japanese Encephalitis Virus (JEV), Chikungunya Virus (CHIKV)), and Human Immunodeficiency Virus-1 (HIV-1). Our analysis also included the embryonic gene PEG10, which represents an established example for -1 PRF in humans ${ }^{43}$. ZAP-S overexpression led to inhibition of - 1PRF for SARS-CoV-1 in vivo by $50 \%$. Among the other frameshift sites we tested, including PEG10, none were responsive to ZAP-S (Fig. 2E). Given the high degree of similarity between the SARS-CoV-1 and CoV-2 frameshift sites, our results demonstrate that the effect of ZAP-S on frameshifting of SARS-CoV is specific. This is unlike the SFL protein, which affects several PRF genes, including the cellular PEG10 3,15.

\section{Impaired viral replication by ZAP-S}

Next, to test that ZAP-S is relevant in the context of infection, Huh7 cells stably overexpressing the ALFAtagged ZAP-S were infected with SARS-CoV-2. We were able to demonstrate that the viral replication was reduced by more than $90 \%$ in cells overexpressing ZAP-S after 24 hours, which was in line with previous reports ${ }^{20,44}$. Confirming our results, recent literature also indicated that siRNA-mediated depletion of ZAP leads to an increase in viral replication ${ }^{20,44}$. Conversely, in our experiments the addition of interferons

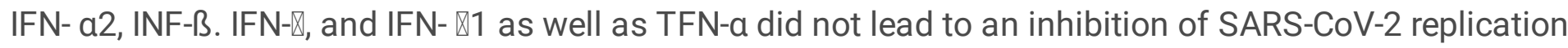
alone or in combination with ZAP-S overexpression (Fig. 2F, Supplementary Fig. 2C) ${ }^{44}$.

Using mutated frameshift elements, it has been reported that even $10 \%$ change in the $-1 \mathrm{frameshift}$ product compared to the in-frame protein product inhibited SARS-CoV viral propagation and reduced infectivity ${ }^{45}$. Furthermore, protein-mediated inhibition of PRF was shown to lead to similar effects on viral infectivity ${ }^{14,46}$. Since ZAP-S levels are upregulated upon infection (Fig. 2D) ${ }^{20,44}$, it is likely that the reduction in viral titers was at least partially due to impaired frameshifting and reduced the -1 frame produced RdRP levels. This is supported by the finding that ZAP-S has a stronger antiviral effect than ZAP-L on SARS-CoV-2 replication ${ }^{20}$. To test that we attempted to quantify the amounts of the frameshift product RdRP (nsp12) in the infected lysates via western blotting and proteomics. However, due to the low sensitivity of the antibody for western blot, and different charges of the frameshift versus nonframeshift products for the MS, so far, we were not able to confidently quantify the level of frameshifted product in infected cells.

\section{ZAP-S decreases SARS-CoV-2 frameshifting efficiency in vitro}


We next focused on characterizing ZAP-S mediated regulation of frameshifting in vitro to dissect the molecular basis for inhibition of - 1PRF. In principle, ZAP-S can either directly interact with the frameshift site or form a complex with auxiliary proteins to modulate translation. To test that, we recombinantly expressed and titrated ZAP-S in the rabbit reticulocyte lysate (RRL) translation system. We employed reporter mRNAs containing nucleotides $12686-14190$ of the SARS-CoV-2 genome to best mimic the native genomic context of viral frameshifting. Control RNAs exclusively producing either the 0 -frame (nsp9-11) or - 1-frame products (nsp9-11 + partial nsp12) were employed as size markers for the western blot (Fig. 3A and 3B). In the absence of ZAP-S, SARS-CoV-2 FE was about 46\% (Fig. 3B), as has been previously reported ${ }^{7}$. With increasing amounts of ZAP-S, there was a corresponding decrease in FE. At the highest concentration of ZAP-S $(3 \mu \mathrm{M}$ ), FE was reduced to 26\% (Fig. 3B and 3C, Supplementary Table 3). To confirm that the observed effect was mediated by ZAP-S and not by random RNA-protein interactions we also analyzed the change in frameshifting upon addition of IGF2BP3 and SUMO-tag alone. IGF2BP3 was identified in our RNA interactome screen, but it was not significantly enriched unlike its close relative IGF2BP1 $\left(\log _{2}\right.$ enrichment $\left.>1.5\right)$, thus rendering it suitable as a control. Here, neither the addition of IGF2BP3, nor the addition of SUMO alone led to a change frameshifting levels

\section{(Supplementary Fig. 2D, E).}

\section{ZAP-S directly interacts with the SARS-CoV-2 frameshifting motif}

In order to dissect the interplay between SARS-CoV-2 frameshift stimulatory RNA structure and ZAP-S in detail, we prepared a series of short synthetic RNAs and we performed RNA-protein binding assays using the highly-sensitive microscale thermophoresis assay (MST). For that, wild-type (WT) RNA containing the - 1PRF signal was derived from nucleotides 13456-13570 of the SARS-CoV-2 genome. The PRF RNA fragment was in vitro transcribed and Cy5-labeled at the 3 ' end to monitor the change in fluorescence in response to ZAP binding.

With the wild-type SARS-CoV-2 pseudoknot, we observed that ZAP-S interaction occurs with the RNA with a high nanomolar affinity $\left(K_{D}=121 \pm 12 \mathrm{nM}\right)$ (Fig. 4A and Supplementary Fig. 3A), which argued that ZAP-S is a direct interaction partner of the frameshift signal. We next employed mutant RNAs to detail the molecular basis of RNA: ZAP-S interaction. The first mutant, SL2 mutant, had a truncation at the 3' end of the putative SARS-CoV-2 pseudoknot, which is predicted to prevent the formation of the putative stem 2 ${ }^{47}$ (Fig. 1A, Fig. 4B). Furthermore, the bulged adenine residue in stem 2 has been shown to be important for SARS-CoV-1 frameshifting ${ }^{37}$. In the SL2 mutant, frameshifting was completely abolished and we detected no interaction with ZAP-S (Fig. 4B, Supplementary Fig. 3B). It was previously suggested that ZAP preferentially binds to CG dinucleotides to discriminate between host and viral RNAs ${ }^{41,48}$. To test whether ZAP-S binding to SARS-frameshift motif was due to the presence of CG motifs, we generated a control RNA with same nucleotide compositions as the wild-type but lacking CG-dinucleotides. In the CGdepleted control RNA, the predicted pseudoknot fold would not form. ZAP-S interaction was also abolished in the CG-depleted control RNA (Fig. 4C and Supplementary Fig. 3C). In addition, we tested the binding of two control proteins, IGF2BP3 and SUMO, to the SARS-CoV-2 frameshift motif. Compared to ZAP-S, IGF2BP3 as a known RBP showed almost 7X lower affinity to the RNA ( $K_{D}=806 \pm 112 \mathrm{nM}$ ). We 
observed no interaction of the SUMO protein with the frameshift element, confirming the specificity of the RNA-binding assay (Supplementary Fig. 3D). Taken together, these data confirm that ZAP-S preferably binds to elements within the frameshift site and support that those direct interactions with the RNA are crucial for translational regulation.

\section{ZAP-S prevents the refolding of the stimulatory RNA}

We next tested whether the interaction between ZAP-S and the - 1PRF RNA alters the RNA structure and mechanical stability. In order to decipher the effect of ZAP-S binding on the folding and unfolding behavior of the frameshifting RNA element, we employed the single-molecule optical-tweezers assay. To this end, a $68 \mathrm{nt}$ long RNA fragment containing the wild type SARS-CoV-2 putative pseudoknot was hybridized to DNA handles. We used the force-ramp method to probe the forces required for unfolding and refolding of the RNA. Briefly, the frameshift RNA was gradually stretched at a constant rate, and then the applied force was released while recording the molecular end-to-end extension distances. This allows the RNA molecule to transition between folded and unfolded states, and sudden changes in measured force-distance trajectories indicate transitions between various RNA conformers (Fig. 4D). With the SARSCoV-2 putative pseudoknot, in the absence of ZAP-S, we observed two major populations. The first one shows a single long step around $20 \mathrm{pN}$ (Fig. 4E, Supplementary Table 5). At decreasing forces, the RNA refolded in two steps, both at about 11 pN (Fig. 4E, Supplementary Fig. 4A, Supplementary Table 5). Such a hysteresis during refolding is commonly reported with pseudoknots and other highly structured RNAs. Moreover, the contour length change obtained by fitting the data with worm-like chain models was $33.5 \pm$ $3.3 \mathrm{~nm}$ (Supplementary Table 5), consistent with the expected value for the full length of the pseudoknot reported previously ${ }^{49,50}$. We also noted the presence of alternative unfolding trajectories, in line with the alternative RNA conformation, which was recently proposed by the Woodside group for SARS-CoV-2 ${ }^{51}$. These trajectories were marked with two consecutive unfolding steps occurring at lower forces around 14 $\mathrm{pN}$ with the contour length change of $15.9 \pm 2.2 \mathrm{~nm}$ (Fig. 4F, Supplementary Fig. 4A, Supplementary Table 5). Regardless of the unfolding trajectory, when the force was released, the RNA refolded back in two steps at around $11 \mathrm{pN}$. Our results were also in line with previous reports on the SARS-CoV-1 frameshift element ${ }^{52}$. We then carried out the same analysis in the presence of the trans-acting protein ZAP-S. To our surprise, in the presence of ZAP-S, the unfolding trajectories of the RNA remained almost unaffected, arguing against an RNA stabilizing effect of the interaction (Fig. 4F, Supplementary Fig. 4B-D, Supplementary Table 5). On the other hand, strikingly refolding of the RNA into its native fold was impaired with less or no detectable transitions into the folded state (Fig. 4F, Supplementary Fig. 4C, D). We thus concluded that ZAP-S interaction impairs the refolding of the SARS-CoV-2 frameshift stimulatory RNA, how this works in the presence of ribosomes awaits investigation.

\section{ZAP-S interacts with translating ribosomes}

Recoding events, in general, are dynamic processes that involve the synergistic action of translating ribosomes and mRNA stimulatory elements. As demonstrated by our ensemble and single-molecule analysis, ZAP-S directly interacts with the SARS-CoV-2 frameshift signal and alters folding of the RNA element. However, several trans-acting factors including the cardiovirus $2 \mathrm{~A}$ and SFL were also shown to 
bind to ribosomes ${ }^{15,53}$. To explore the possibility of whether ZAP also interacts with ribosomes during translation, we performed ribosome pelleting assay (Fig. 5A). We employed lysates of SARS-CoV-2 infected and uninfected Huh7 cells stably overexpressing ZAP-S. Ribosomes in these lysates were then pelleted through a sucrose cushion, and associated proteins were analyzed via western blotting (Fig. 5B). We were able to demonstrate that ZAP-S was present in the ribosome pellet. Since ZAP-L is constitutively expressed in human tissues, we also detected the presence of endogenous ZAP-L in the ZAP-S overexpressed cells ${ }^{33}$. To test whether the endogenous ZAP-S is also associated with ribosomes, we similarly processed infected and uninfected naïve Calu-3 cells without any ZAP overexpression (Supplementary Fig. 5A). Likewise, endogenous ZAP-S and ZAP-L could also be easily detected in the ribosome pellet. Additionally, we also detected viral nsp1 protein in the pellet, which is known to associate with the small ribosomal subunit during SARS-CoV-2 infection (Supplementary Fig. 5A) ${ }^{54}$.

To test whether ZAP-S is associated with actively translating ribosomes, we also performed polysome profiling using HEK293 cells overexpressing ZAP-S. ZAP-S was detected in most of the ribosomal fractions including polysomes (Fig. 5 C). In contrast, the endogenous longer isoform, ZAP-L was identified only in the free RNA fractions. We observed similar polysome profiles with cells overexpressing the SFL, which was bound to the free RNA, the 40S and 60S subunits, the 80S ribosomes, and polysomes (Supplementary Fig. 5B) ${ }^{15}$. Collectively, these results indicate that ZAP-S can be found associated with ribosomes, including the actively translating polysomes. Therefore, we posited the involvement of ZAP in fine-tuning translation. It remains to be investigated, whether this is a physiological phenomenon occurring during different viral infections.

We were surprised to observe strong association of ZAP with RNA and ribosomes, which may provide some clues on how the factor can regulate translation of viral RNAs. To detail physiologically relevant cellular interaction partners of ZAP during infections, we performed co-immunoprecipitation (co-IP) in SARS-CoV-2 infected Calu-3 cells. Captured proteins following affinity purification were detected by LCMS/MS analysis (Supplementary Fig. 5C, D, Supplementary Table 2). As a control to differentiate between RNA- vs. protein-mediated interactions, we also treated the lysates with Benzonase endonuclease, which degrades nucleic acids. Upon analysis, a total of 124 proteins were detected with at least 4-fold enrichment. The prominence of translation-relevant proteins in untreated and nuclease treated samples was striking. $41 \%$ of the enriched proteins were reported to be involved in translation, $48 \%$ in protein localization to the ER, $44 \%$ in translation initiation, and $41 \%$ in ribosome biogenesis (Supplementary Fig. 5C). Previously it was reported that ZAP interacts with proteins including TRIM25 ${ }^{39}$, elF4A ${ }^{55}$ and KHNYN ${ }^{40}$. In the present co-IP analysis, we were able to detect TRIM21 - a close relative of TRIM25, which supports the interplay between TRIM proteins and ZAP ${ }^{56}$. We also identified elF4H as a prominent hit in our analysis, which is the cofactor of the DEAD-box RNA helicase elF4A ${ }^{57}$. Furthermore, we detected the RNA-binding protein LARP1 as a ZAP-interaction partner, which may point to a novel route for regulation of translation initiation by ZAP ${ }^{58}$. As for ribosomal proteins, we found large subunit proteins RPLP0, RPLP2, RPL10A, RPL12, RPS12 and RPS16 among the most enriched interaction partners (Fig. 5D). Intriguingly, in contrast to SFL, which stimulated premature termination, we were not 
able to detect any translation release factors, pointing to a crucial difference in their mechanisms of action. Taken together, these results indicate that ZAP does not only associate with RNA elements but also with ribosomal proteins through protein-protein mediated interactions. This expands the role of ZAP as a SARS-CoV frameshifting modulator, proposing novel functions beyond its previously suggested roles in inhibition of translation initiation ${ }^{55}$. Currently, we are further dissecting these interactions using structural analysis tools.

\section{Discussion}

Programmed ribosomal frameshifting (-1PRF) is indispensable for the expression of RNA-dependent RNA polymerase (RdRP) of coronaviruses. In this study, we explored for the first time whether transfactors modulate SARS-CoV-2 - 1PRF in infected versus uninfected cells. We discovered that the interferon-induced antiviral protein ZAP-S can strongly impair SARS-CoV-2 frameshifting.

A large number of the interactors found in our screen are well-known RNA-binding proteins and/or regulators of translation. Most of the interactors we identified using the minimal frameshift element as the bait also appeared in genome-wide studies of SARS-CoV-2 RNA binding proteins ${ }^{3,30-32}$. Despite technical differences and different systems employed among these studies, ZAP-S was surprisingly one of the prominent common hits. We observed that many of the binders were not changing the frameshift levels in vitro, which is in line with the notion that mRNA association alone is not sufficient to modulate frameshifting levels. In addition, the mechanism by which ZAP acts on the frameshifting seems to be more complex than anticipated for other cis-and trans-acting regulators of frameshifting. It is well established that ZAP can exert its antiviral activity by binding to CG-rich RNAs and thereby targeting them for degradation. Yet, ZAP does not bind to all CG-rich sequences and location of these sequences were shown to be crucial for ZAP interaction ${ }^{36,37}$. We speculate that, in addition to recognizing CG dinucleotides within the SARS-CoV-2 RNA frameshift site, ZAP-S also recognizes a particular fold within the putative pseudoknot. This way the factor modulates RNA folding pathways and thus plasticity, which is a common feature of RNA-based regulation of biological processes ${ }^{59,60}$. In fact, structural plasticity has been reported as a hallmark of - 1PRF stimulatory RNAs ${ }^{47}$. Our single-molecule pulling experiments indicate that ZAP-S binds to the SARS-CoV-2 - 1PRF RNA directly and interferes with the refolding of the stimulatory RNA structure in vitro. As a result, unlike other viral and cellular factors, such as the cardiovirus $2 \mathrm{~A}$ or poly $(\mathrm{C})$ binding protein, which were shown to directly associate with RNA motifs to induce frameshifting, here association ZAP-S, a host-encoded antiviral protein with the RNA element significantly reduces frameshifting ${ }^{12,14}$.

Beyond solely binding the - 1PRF stimulatory structure, we were surprised to detect interactions between ZAP-S and the translation machinery. The translation-inhibitory effect of ZAP was previously suggested to be limited to initiation through sequestration of elF4A. The repression of translation by ZAP was also reported to be stimulated by its co-factor TRIM $25^{39}$. In our in vitro assays, ZAP-S alone was sufficient to modulate translational frameshifting without additional co-factors. Interaction with ribosomes was 
observed also with other trans-factors such as the SFL protein ${ }^{15}$. However, despite the similarities, our data suggests a different mechanism of action for ZAP-S. SFL was shown to interact with stalled ribosomes and recruit release factors to terminate translation, and it acts not only on the HIV- 1 but also other frameshift sites including the cellular frameshift gene PEG10 ${ }^{15}$. Here, we were not able to detect any release factors by ZAP co-IP analysis, and effect of ZAP-S was specific to SARS-CoV-2 and the closely related SARS-CoV-1. Overall, these findings establish ZAP-S as the first cellular factor, which has a direct role in modulating SARS-coronavirus frameshifting. In accordance with previously published results, we demonstrate that overexpression of ZAP-S reduces the replication of SARS-CoV- 2 by $90 \%{ }^{44}$, ${ }^{20}$. Further studies are required to deconvolute the multivalent effects of ZAP-S on immunity, viral replication and RNA-related processes such as translation and RNA stability $33,38-42$.

Ultimately, based on our findings, we propose the following model for the inhibition of - 1PRF by ZAP-S (Fig. 6). Accordingly, ZAP-S binding to the frameshift RNA can alter the stimulatory RNA structure and reduce the chance of elongating ribosomes to encounter the stimulatory pseudoknot. Without a stimulatory structure, the elongation pause during next round of translation would be too short for codonanti-codon interactions to be established in the -1 frame. Thus, ZAP-S would likely allow translation to proceed without any roadblock effect and terminate at the 0 -frame UAA stop codon found immediately downstream of the slippery sequence. How exactly the binding of ZAP-S and other riboregulators to ribosomes would impact kinetics of translation and frameshifting awaits further investigation.

Given the plethora of mechanisms presented by which trans-regulators of PRF can act, it is conceivable that viral and host encoded trans-factors follow a multitude of routes to impact frameshift paradigms. Taken together, this study highlights a novel role for ZAP-S in SARS-CoV-2 antiviral response and establishes ZAP-S as a bona fide regulator of SARS-CoV-2 translation. This work also provokes the idea of using ZAP-S as a potential SARS-CoV-2 intervention strategy, by employing ZAP-S mRNA as a multifunctional antiviral and immune-modulatory drug.

\section{Materials And Methods}

\section{RNA affinity pulldown mass spectrometry}

RNA antisense purification was performed according to a protocol based on ${ }^{17}$. Briefly, $6{ }^{\star} 10^{\wedge} 7 \mathrm{HEK} 293$ cells per condition were lysed in a buffer containing $20 \mathrm{mM}$ Tris/ $\mathrm{HCl} \mathrm{pH} \mathrm{7.5,} 100 \mathrm{mM} \mathrm{KCl}, 5 \mathrm{mM} \mathrm{MgCl} 2,1$ mM DTT, 0.5 \% Igepal CA630 (Sigma-Aldrich), 1× c0mplete ${ }^{\text {TM }}$ Protease Inhibitor Cocktail (Roche), $40 \mathrm{U} / \mathrm{ml}$ RNase inhibitor (Molox). The cleared lysate was incubated with in vitro transcribed RNA corresponding to the SARS-CoV-2 - 1PRF site, which was immobilized on streptavidin hydrophilic magnetic beads (NEB) by biotin-streptavidin interaction. After three washes with binding buffer (50 mM HEPES/KOH pH 7.5, 100 $\mathrm{mM} \mathrm{NaCl}, 10 \mathrm{mM} \mathrm{MgCl}$ ) and two washes with wash buffer (50 mM HEPES/KOH pH 7.5, $250 \mathrm{mM} \mathrm{NaCl}$, $10 \mathrm{mM} \mathrm{MgCl} 2$ ), bound proteins were eluted by boiling the sample in $1 \times$ NuPAGE LDS sample buffer (Thermo Fisher Scientific) supplemented with 40 mM DTT. For infected as well as uninfected Calu-3 cells 
the procedure was performed similarly. In order to inactivate the virus, the lysis buffer contained Triton$\mathrm{X} 100$ and inactivation was confirmed by plaque assays.

For LC-MS/MS, the eluted proteins were alkylated using iodoacetamide followed by acetone precipitation. In solution digests were performed in $100 \mathrm{mM}$ ammonium bicarbonate and $6 \mathrm{M}$ urea using Lys-C and after reducing the urea concentration to $4 \mathrm{M}$ with trypsin. Peptides were desalted using $\mathrm{C} 18$ stage tips and lyophilized. LC-MS/MS was performed at the RVZ Proteomics Facility (Würzburg) and analyzed as described previously ${ }^{61}$. Gene ontology (GO) term analysis was performed with Panther ${ }^{62}$. The list of all identified proteins is given in Supplementary Table 1.

\section{Co-immunoprecipitation}

Endogenous interaction partners of ZAP were identified by co-immunoprecipitation followed by mass spectrometry as published previously ${ }^{63}$. Briefly, uninfected and SARS-CoV-2-infected Calu-3 cells were lysed in lysis buffer (10 mM Tris/HCl pH 7.4, $150 \mathrm{mM} \mathrm{NaCl}, 1 \%$ Igepal CA630 (Sigma-Aldrich) and 1x cOmplete ${ }^{\mathrm{TM}}$ Protease Inhibitor Cocktail (Roche)). The lysis buffer was supplemented either with $40 \mathrm{U} / \mathrm{ml}$ RNase inhibitor (Molox) or $50 \mathrm{U} / \mathrm{ml}$ Benzonase (Roche) to differentiate between RNA- and proteindependent interactions. $1 \mathrm{mg}$ of cell lysate was cleared with protein A magnetic beads to remove nonspecific interactions (S1425S, NEB) and incubated overnight with anti-ZAP antibody (Proteintech 168201-AP) or anti-IgG from rabbit as a control (Cell Signaling, a gift from Dr. Mathias Munschauer, HIRI-HZI). Antibodies were captured with protein A magnetic beads, washed with lysis buffer, and eluted by boiling in $1 X_{\text {NuPAGE }}^{\text {TM }}$ LDS Sample Buffer (Thermo Fisher Scientific) supplemented with 40 mM DTT. LCMS/MS proceeded as described above. A list of all identified proteins can be found in Supplementary

\section{Table 2.}

\section{Plasmid construction}

To generate dual-fluorescence reporter constructs frameshift sites of SARS-CoV, SARS-CoV-2, MERS-CoV, BtCoV 273, HIV-1, JEV, PEG10, WNV were placed between the coding sequence of EGFP and mCherry (parental construct was a gift from Andrea Musacchio (Addgene plasmid \# $87803{ }^{64}$ ) by site-directed mutagenesis in a way that EGFP would be produced in 0 -frame and mCherry in - 1-frame. EGFP and mCherry were separated by StopGo ${ }^{65}$ signals as well as an alpha-helical linker ${ }^{66}$. A construct with no PRF insert and mCherry in-frame with EGFP served as a $100 \%$ translation control and was used to normalize EGFP and mCherry intensities.

To generate screening vectors, protein-coding sequences of DDX17 (NM_001098504.2), DDX36 (NM_020865.3), ELAVL1 (NM_001419.3), GNL2 (NM_013285.3), HNRNPF (NM_001098204.2), HNRNPH1 (NM_001364255.2), HNRNPH2 (NM_001032393.2), IGF2BP1 (NM_006546.4), MATR3 iso 2 (NM_018834.6), MMTAG2 (NM_024319.4), NAF1 (NM_138386.3), NHP2 (NM_017838.4), POP1 (NM_001145860.2), RAP11B (NM_004218.4), RSL1D1 (NM_015659.3), SFL (NM_018381.4), SURF6 (NM_001278942.2), TFRC (NM_003234.4), ZAP (NM_024625.4) and ZNF346 (NM_012279.4) were placed in frame with the coding sequence for ECFP in pFlp-Bac-to-Mam (gift from Dr. Joop van den Heuvel, HZI, Braunschweig, Germany ${ }^{67}$ ) via Gibson Assembly 68. 
Golden Gate compatible vectors for heterologous overexpression in E. coli, in vitro translation in RRL, and lentivirus production, were generated by Golden Gate or Gibson Assembly. A dropout cassette was included to facilitate the screening of positive colonies. Protein-coding sequences were introduced by Golden Gate Assembly using Aarl cut sites ${ }^{69}$. pET-SUMO-GFP (gift from Prof. Utz Fischer, JuliusMaximilians-University, Würzburg, Germany) was used as the parental vectors for protein overexpression in E. coli. The lentivirus plasmid was a gift from Prof. Chase Beisel (HIRI-HZI, Würzburg, Germany). An ALFA-tag was included to facilitate the detection of the expressed protein ${ }^{70}$. The frameshift reporter vector for the in vitro translation contained $B$-globin 5 ' and $3^{\prime}$ UTRs as well as a $30 \mathrm{nt}$ long poly(A) tail. The insert was derived from nucleotides 12686-14190 of SARS-CoV-2 (NC_045512.2); a 3×FLAG-tag was introduced at the $\mathrm{N}$-terminus to facilitate detection. To generate $0 \%$ and $100 \%-1 \mathrm{PRF}$ controls, the $-1 \mathrm{PRF}$ site was mutated by disrupting the pseudoknot structure as well as the slippery sequence.

Optical tweezers constructs were based on the wild type SARS-CoV-2 frameshifting site (nucleotides 13475-13541) cloned into the plasmid pMZ_lambda_OT, which encodes for the optical tweezer handle sequences (2Kb each) flanking the RNA structure (130 nt). Constructs were generated using Gibson Assembly. Sequences of all plasmids and oligos used in this study are given in Supplementary Table 4.

\section{Cell culture, transfections, generation of polyclonal stable cell lines}

HEK293 cells (gift from Prof. Jörg Vogel, HIRI-HZI) and Huh7 cells (gift from Dr. Mathias Munschauer, HIRI-HZI), were maintained in DMEM (Gibco) supplemented with 10\% FBS (Gibco) and $100 \mathrm{U} / \mathrm{ml}$ streptomycin and $100 \mathrm{mg} / \mathrm{ml}$ penicillin. Calu-3 cells (ATCC HTB-55) were cultured in MEM (Sigma) supplemented with $10 \% \mathrm{FBS}$. Cell lines were kept at $37^{\circ} \mathrm{C}$ with $5 \% \mathrm{CO} 2$. Transfections were performed using PEI (Polysciences) according to manufacturer's instructions. For co-transfections, plasmids were mixed at a 1:1 ratio.

VSV-G envelope pseudo-typed lentivirus for the generation of stable cell lines was produced by cotransfection of each transfer plasmid with pCMVdR $8.91^{71}$ and pCMV-VSV-G (gift from Prof. Weinberg, Addgene plasmid \# $8454{ }^{72}$ ). 72 h post-transfection, the supernatant was cleared by centrifugation and filtration. The supernatant was used to transduce naïve Huh7 cells in the presence of $10 \mu \mathrm{g} / \mathrm{ml}$ polybrene (Merck Millipore). After $72 \mathrm{~h}$, the cells were selected with $10 \mu \mathrm{g} / \mathrm{ml}$ blasticidin (Cayman Chemical) for 10 days to generate polyclonal cell lines.

\section{SARS-CoV-2 infection}

For infection with SARS-CoV-2, we used the strain hCoV-19/Croatia/ZG-297-20/2020, a kind gift of Prof. Alemka Markotic (University Hospital for Infectious Diseases, Zagreb, Croatia). The virus was raised for two passages on Caco-2 cells (HZI Braunschweig). Calu-3 cells (ATCC HTB-55) were infected with 2000 $\mathrm{PFU} / \mathrm{ml}$ corresponding to an $\mathrm{MOI}$ of 0.03 at $24 \mathrm{~h}$ post-infection, cells were collected and lysed for proteomic and ribosome-interaction experiments. To study the effect of ZAP-S on SARS-CoV-2 infection, Huh-7 cells were employed. One hour before infection, Huh-7 cells both naïve or ZAP-S-overexpressing

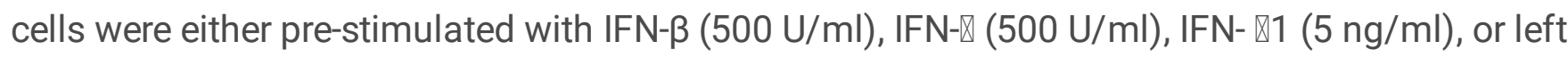
untreated. Cells were infected with 200000 pfu/ml, corresponding to an $\mathrm{MOI}$ of 0.03 at 24h post-infection, 
cell culture supernatants were collected and titrated by plaque assay on Vero E6 cells (ATCC CRL-1586). Briefly, confluent Vero E6 cells in 96-well plates were inoculated with dilutions of the virus-containing supernatants for one hour at $37^{\circ} \mathrm{C}$, the inoculum was removed and cells were overlaid with MEM containing $1.75 \%$ methyl-cellulose. At three days post-infection, whole wells of the plates were imaged using an IncuCyte S3 (Sartorius) at 4x magnification, and plaques were counted visually.

\section{Flow cytometry}

HEK293 cells were transiently transfected with either the control construct or the - 1PRF construct encoding for the dual-fluorescence EGFP-mCherry translation reporter as outlined in Fig. 2A. Cells were harvested at $24 \mathrm{~h}$ post-transfection and fixed with $0.4 \%$ formaldehyde in PBS. After washing with PBS, flow cytometry was performed on a FACSAria III (BD Biosciences) or a NovoCyte Quanteon (ACEA) instrument. Flow cytometry data were analyzed with FlowJo software (BD Biosciences). ECFP-positive cells were analyzed for the ratio between mCherry and EGFP. FE was calculated according to the following formula:

$$
\mathrm{FE}(\%)=\frac{m \text { Cherrytest } / E G P_{\text {test }}}{m \text { Cherrycontrol } / E G F P_{\text {test }}}
$$

where mCherry represents the mean mCherry intensity, EGFP the mean EGFP intensity, test represent the tested sample and control represents the in-frame control where mCherry and EGFP are produced in an equimolar ratio ${ }^{73}$. Data represent the results of at least three independent experiments.

\section{Purification of recombinant proteins}

Recombinant ZAP-S N-terminally tagged with $6 \times$ His-SUMO was purified from $E$. coli Rosetta 2 cells (Merck) by induction with $0.2 \mathrm{mM}$ isopropyl $\beta$-d-1-thiogalactopyranoside for $18 \mathrm{~h}$ at $18^{\circ} \mathrm{C}$. Cells were collected, resuspended in lysis buffer (50 mM HEPES/KOH pH 7.6, $1 \mathrm{M} \mathrm{NaCl}, 1 \mathrm{mM}$ DTT, 1 mM PMSF) and lysed in a pressure cell. The lysate was cleared by centrifugation and ZAP-S was captured using NiNTA resin (Macherey-Nagel). After elution with $500 \mathrm{mM}$ imidazole, ZAP-S was further purified and the bound nucleic acids removed by size exclusion chromatography (HiLoad ${ }^{\circledR} 16 / 600$ Superdex $\circledast 200$ ) in 20 $\mathrm{mM} \mathrm{HEPES/KOH} \mathrm{pH} \mathrm{7.6,1} \mathrm{M} \mathrm{KCl,} 1$ mM DTT, 20\% glycerol. Protein identity was verified by SDS-PAGE as well as western blotting (Supplementary Fig. 2D). Purified ZAP-S was rapidly frozen and stored in aliquots at $-80^{\circ} \mathrm{C}$.

His-SUMO-IGF2BP3 as well as His-SUMO were kind gifts from Dr. Andreas Schlundt (Goethe University, Frankfurt, Germany)

\section{Western blots}

Protein samples were denatured at $95^{\circ} \mathrm{C}$ and resolved by $12 \%$ SDS-PAGE at $30 \mathrm{~mA}$ for $2 \mathrm{~h}$. After transfer using Trans-Blot (Bio-Rad), nitrocellulose membranes were developed using the following primary antibodies: anti-His-tag (ab18184), anti-DDDDK (ab49763), anti-ALFA (FluoTag®-X2 anti-ALFA AlexaFluor 
647), anti-ZC3HAV1 (Proteintech 16820-1-AP). The following secondary antibodies were used: IRDye ${ }^{\circledR}$ $800 \mathrm{CW}$ Goat anti-rabbit and IRDye ${ }^{\circledR}$ 680RD Donkey anti-Mouse (both LI-COR). Bands were visualized using an Odyssey Clx infrared imager system (LI-COR) or a Typhoon7000 (GE Healthcare).

\section{In vitro translation assays}

mRNAs were in vitro transcribed using T7 polymerase purified in-house using linearized plasmid DNA as the template. These mRNAs were capped (Vaccinia Capping System, NEB) and translated using the nuclease-treated rabbit reticulocyte lysate (RRL; Promega). Typical reactions were comprised of $75 \% \mathrm{v} / \mathrm{v}$ $\mathrm{RRL}, 20 \mu \mathrm{M}$ amino acids, and were programmed with $\sim 50 \mu \mathrm{g} / \mathrm{ml}$ template mRNA. ZAP-S was titrated in the range of $0-3 \mu \mathrm{M}$. Reactions were incubated for $1 \mathrm{~h}$ at $30^{\circ} \mathrm{C}$. Samples were mixed with $3 \mathrm{X}$ volumes of 1X NuPAGE ${ }^{\text {TM }}$ LDS Sample Buffer (Invitrogen), boiled for 3 min, and resolved on a NuPAGE ${ }^{\text {TM }} 4$ to $12 \%$ BisTris polyacrylamide gel (Invitrogen). The products were detected using western blot (method as described above). The nitrocellulose membranes were developed using anti-DDDDK primary (Abcam ab49763) and IRDye ${ }^{\circledR}$ 680RD donkey anti-mouse secondary antibody (LI-COR). Bands were visualized using an Odyssey Clx infrared imager system (LI-COR). Bands corresponding to the -1 or 0 -frame products, 58 $\mathrm{kDa}$ and $33 \mathrm{kDa}$ respectively, on western blots of in vitro translations were quantified densitometrically using Image J software ${ }^{74}$. FE was calculated as previously described, by the formula intensity (-1frame)/ (intensity (-1-frame) + intensity (0-frame) $)^{11}$. The change in FE was calculated as a ratio of FE of each condition to the FE of no-protein control in each measurement. Experiments were repeated at least 3 independent times.

\section{Microscale thermophoresis}

Short frameshifting RNA constructs were in vitro transcribed using T7 polymerase as described above. RNAs were labeled at the 3' end using pCp-Cy5 (Cytidine-5'-phosphate-3'-(6-aminohexyl) phosphate) (Jena Biosciences). For each binding experiment, RNA was diluted to $10 \mathrm{nM}$ in Buffer A (50 mM Tris- $\mathrm{HCl}$ $\mathrm{pH} 7.6,250 \mathrm{mM} \mathrm{KCl}, 5 \mathrm{mM} \mathrm{MgCl} 2,1 \mathrm{mM} \mathrm{DTT}, 5 \%$ glycerol supplemented with $0.05 \%$ Tween 20 and 0.2 $\mathrm{mg} / \mathrm{ml}$ E. coli tRNA). A series of 16 tubes with ZAP-S dilutions were prepared in Buffer A on ice, producing ZAP-S ligand concentrations ranging from $40 \mathrm{pM}$ to $2 \mu \mathrm{M}$. For measurements, each ligand dilution was mixed with one volume of labeled RNA, which led to a final concentration of $5.0 \mathrm{nM}$ labeled RNA. The reaction was mixed by pipetting, incubated for $10 \mathrm{~min}$ at room temperature, followed by centrifugation at $10,000 \times \mathrm{g}$ for $5 \mathrm{~min}$. Capillary forces were used to load the samples into Monolith NT.115 Premium Capillaries (NanoTemper Technologies). Measurements were performed using a Monolith Pico instrument (NanoTemper Technologies) at an ambient temperature of $25^{\circ} \mathrm{C}$. Instrument parameters were adjusted to $5 \%$ LED power, medium MST power, and MST on-time of 5 seconds. An initial fluorescence scan was performed across the capillaries to determine the sample quality and afterward, 16 subsequent thermophoresis measurements were performed. Data of three independently pipetted measurements were analyzed for the $\Delta$ Fnorm values, and binding affinities were determined by the MO. Affinity Analysis software (NanoTemper Technologies). Graphs were plotted using GraphPad Prism 8.4.3 software.

\section{Microscopy}

Page 15/31 
HEK293 cells were cultured on glass slides and transfected as described above. The cells were fixed with $4 \%$ paraformaldehyde in $1 \times$ PBS for 15 min at room temperature. After washing with $1 \times$ PBS, cells were mounted in ProLong Antifade Diamond without DAPI (Invitrogen). Microscopy was performed using a Thunder Imaging System (Leica) using 40\% LED power and the 40x objective. EGFP was excited at 460$500 \mathrm{~nm}$ and detected at 512-542 nm. mCherry was excited at 540-580 nm and detected at 592-668 $\mathrm{nm}$. The images were processed with the LasX software (Leica).

\section{Polysome profiling analysis}

A plasmid expressing ZAP-S N-terminally tagged with a His-tag was transfected into HEK293 cells using $\mathrm{PEl}$, as described above. To check endogenous ZAP-S expression, HEK cells were transfected with a plasmid containing the same backbone and His-tag. At $24 \mathrm{~h}$ post-transfection, cycloheximide (VWR) was added to the medium at a final concentration of $100 \mu \mathrm{g} / \mathrm{ml}$ to stop translation. Approximately $107 \mathrm{HEK}$ cells were lysed with $500 \mu$ lysis buffer $(20 \mathrm{mM}$ Tris- $\mathrm{HCl} \mathrm{pH} \mathrm{7.4,} 150 \mathrm{mM} \mathrm{NaCl}, 5 \mathrm{mM} \mathrm{MgCl} 2,1 \mathrm{mM}$ DTT, $100 \mu \mathrm{g} / \mathrm{ml}$ Cycloheximide, $1 \%$ Triton X), and the lysate was clarified by centrifugation at 17,0000 $\times \mathrm{g}$ for $10 \mathrm{~min}$ at $4^{\circ} \mathrm{C}$. Polysome buffer $(20 \mathrm{mM}$ Tris- $\mathrm{HCl} \mathrm{pH} \mathrm{7.4,} 150 \mathrm{mM} \mathrm{NaCl}, 5 \mathrm{mM} \mathrm{MgCl} 2,1 \mathrm{mM}$ DTT, 100 $\mu \mathrm{g} / \mathrm{ml}$ Cycloheximide) was used to prepare all sucrose solutions. Sucrose density gradients $(5-45 \% \mathrm{w} / \mathrm{v})$ were freshly made in SW41 ultracentrifuge tubes (Beckman) using a Gradient Master (BioComp Instruments) according to manufacturer's instructions. The lysate was then applied to a $4-45 \%$ sucrose continuous gradient and centrifuged at 35,000 rpm (Beckmann Coulter Optima XPN) for $3 \mathrm{~h}$, at $4^{\circ} \mathrm{C}$. The absorbance at $254 \mathrm{~nm}$ was monitored and recorded and $500 \mu \mathrm{l}$ fractions were collected using a gradient collector (BioComp instruments). The protein in each fraction was pelleted with trichloroacetic acid, washed with acetone, and subjected to western blotting, as described above.

\section{Ribosome pelleting assay}

SARS-CoV-2 Calu-3 infected lysates were prepared as described above. $300 \mu \mathrm{l}$ of the lysate was loaded onto a $900 \mu \mathrm{l} 1 \mathrm{M}$ sucrose cushion in polysome buffer (described above) in Beckman centrifugation tubes. Ribosomes were pelleted by centrifugation at 75,000 rpm for $2 \mathrm{~h}$, at $4^{\circ} \mathrm{C}$, using a Beckmann MLA130 rotor (Beckman Coulter Optima MAX-XP). After removing the supernatant, ribosome pellets were resuspended in polysome buffer and was used for western blotting, as described above.

\section{Optical tweezers constructs}

$5^{\prime}$ and 3' DNA handles, and the template for in vitro transcription of the SARS-CoV-2 putative pseudoknot RNA were generated by PCR using the pMZ_lambda_OT vector. The 3 ' handle was labeled during the PCR using a $5^{\prime}$ digoxigenin-labeled reverse primer. The $5^{\prime}$ handle was labeled with Biotin-16-dUTP at the $3^{\prime}$ end following PCR using T4 DNA polymerase. The RNA was in vitro transcribed using T7 RNA polymerase. Next, DNA handles ( $5^{\prime}$ and $\left.3^{\prime}\right)$ and in vitro transcribed RNA were annealed in a mass ratio 1:1:1 (5 $\mu \mathrm{g}$ each) by incubation at $95^{\circ} \mathrm{C}$ for $10 \mathrm{~min}, 62^{\circ} \mathrm{C}$ for $2 \mathrm{~h}, 52^{\circ} \mathrm{C}$ for $2 \mathrm{~h}$ and slow cooling to $4^{\circ} \mathrm{C}$ in annealing buffer ( $80 \%$ formamide, $400 \mathrm{mM} \mathrm{NaCl}, 40 \mathrm{mM}$ HEPES, pH 7.5, and $1 \mathrm{mM}$ EDTA, pH 8) to yield the optical tweezer suitable construct (Fig. 4E). Following the annealing, samples were concentrated by ethanol precipitation, pellets were resuspended in $40 \mu \mathrm{l}$ RNase-free water, and $4 \mu \mathrm{l}$ aliquots were stored at $-20^{\circ} \mathrm{C}$ until use. 


\section{Optical tweezers data collection and analysis}

Optical tweezers measurements were performed using a commercial dual-trap platform coupled with a microfluidics system (C-trap, Lumicks). For the experiments, optical tweezers (OT) constructs were mixed with $4 \mu \mathrm{l}$ of polystyrene beads coated with antibodies against digoxigenin (AD beads, $0.1 \% \mathrm{~V} / \mathrm{v}$ suspension, $\varnothing 1.76 \mu \mathrm{m}$, Spherotech), $10 \mu$ of assay buffer (20 mM HEPES, pH 7.6, $300 \mathrm{mM} \mathrm{KCl,} 5 \mathrm{mM}$ $\mathrm{MgCl} 2,5 \mathrm{mM}$ DTT and $0.05 \%$ Tween) and $1 \mu \mathrm{l}$ of RNase inhibitor. The mixture was incubated for $20 \mathrm{~min}$ at room temperature in a final volume of $19 \mu \mathrm{l}$ and subsequently diluted by the addition of $0.5 \mathrm{ml}$ assay buffer. Separately, $0.8 \mu \mathrm{l}$ of streptavidin-coated polystyrene beads (SA beads, $1 \% \mathrm{v} / \mathrm{v}$ suspension, $\varnothing 2 \mu \mathrm{m}$, Spherotech) were mixed with $1 \mathrm{ml}$ of assay buffer. The flow cell was washed with the assay buffer, and suspensions of both streptavidin beads and the complex of OT construct with anti-digoxigenin beads were introduced into the flow cell. During the experiment, an anti-digoxigenin (AD) bead and a streptavidin (SA) bead were trapped and brought into proximity to allow the formation of a tether. The beads were moved apart (unfolding) and back together (refolding) at a constant speed $(0.05 \mu \mathrm{m} / \mathrm{s})$ to yield the forcedistance (FD) curves. The stiffness was maintained at 0.31 and $0.24 \mathrm{pN} / \mathrm{nm}$ for trap 1 (AD bead) and trap 2 (SA bead), respectively. For experiments with ZAP-S protein, recombinantly expressed ZAP-S was diluted to $400 \mathrm{nM}$ in assay buffer and introduced to the flow cell. FD data were recorded at a rate of $78125 \mathrm{~Hz}$.

Raw data files were processed using our custom-written python algorithm called Practical Optical Tweezers Analysis TOol (POTATO, https://github.com/lpekarek/POTATO.git, manuscript in preparation). In brief, raw data were first down sampled by a factor of 20 to speed up subsequent processing, and the noise was filtered using Butterworth filter ( 0.05 filtering frequency, filter order 2 ). Numerical time derivation was calculated separately for force and distance data. These derivations were statistically analyzed to identify the folding events and their coordinates. For data fitting, we employed a combination of two worm-like chain models (WLC1 for the fully folded double-stranded parts and WLC2 for the unfolded single-stranded parts) as described previously ${ }^{53}$. Firstly, the initial contour length of the folded RNA was set to $1231 \mathrm{~nm}$, and the persistence length of the double-stranded part was fitted ${ }^{53}$. Then, the persistence length of the unfolded RNA was set to $1 \mathrm{~nm}$, and the contour length of the single-stranded part was fitted. Data were statistically analyzed, and the results were plotted using Prism 8.0.2 (GraphPad).

\section{qRT-PCR}

Total RNA was isolated as described previously ${ }^{75}$, and the reverse transcription using RevertAid (Invitrogen) was primed by oligo(dT). Reactions of quantitative real-time PCR (qRT-PCR) were set up using POWER SYBR green Master-mix (Invitrogen) according to manufacturer's instructions and analyzed on the CFX96 Touch Real-Time PCR Detection System (Bio-Rad) under the following cycling condition: $50^{\circ} \mathrm{C}$ for $2 \mathrm{~min}, 95^{\circ} \mathrm{C}$ for $2 \mathrm{~min}$, followed by 40 cycles of $95^{\circ} \mathrm{C}$ for $15 \mathrm{~s}$ and $60^{\circ} \mathrm{C}$ for $30 \mathrm{~s}$, and ending with a melt profile analysis. The fold change in mRNA expression was determined using the 2- $\Delta \Delta \mathrm{Ct}$ method relative to the values in uninfected samples, after normalization to the housekeeping gene (geometric mean) GAPDH. Statistical analysis was conducted using an unpaired two-tailed $t$-test with Welch's 
correction comparing delta $\mathrm{Ct}$ values of the respective RNA in uninfected and infected cells. The results were plotted using Prism 8.0.2 (GraphPad).

\section{Quantification and statistical analysis}

All statistical analyses and software used have been mentioned in the Figure Legends and Materials \& Methods. Measurements from the in vitro western blot assay and in vivo dual fluorescence assay resulted from 3 technical replicates. Measurements from single-molecule experiments resulted from a specified number ( $\mathrm{n}$ ) of traces from a single experiment. For the ensemble MST analysis, all analysis from 3 individual replicates was performed in Nanotemper MO. Affinity software.

\section{Declarations}

\section{Acknowledgments}

We thank Dr. Zeljka Macak-Safranko and Prof. Alemka Markotic (University of Zagreb) for providing the SARS-CoV-2 virus isolate prior to publication. We thank Dr. Andreas Schlundt for kind gifts of IGF2BP3 and SUMO proteins (Goethe University, Frankfurt, Germany). We thank Dr. Joop van den Heuvel (HZI) for his suggestions on ZAP-S purification. We thank Prof. Redmond Smyth, Prof. Jörg Vogel, Prof. Lars Dölken, Prof. Utz Fischer and Prof. Thomas Pietschmann for critical reading of the manuscript. We thank expert technical assistance by Tatyana Koch (HIRI-HZI). We thank Stefan Buck for help with optical tweezer data acquisition. We thank Ayse Barut for cell maintenance for infection studies (HZI). We thank Dr. Andreas Schlosser and Stephanie Lamer from the Rudolf Virchow Center for the LC-MS/MS analysis. Figures were partially generated using BioRender.com.

\section{Funding}

This project is funded fully or in part by the

Helmholtz Association

MWK Niedersachsen through Grant Nr. 14-76103-184 CORONA-2/20.

NC received funding from the European Research Council (ERC) Grant Nr. 948636.

\section{Author contributions}

Conceptualization: MZ, AK, NC

Methodology: MZ, AK, NC, UR, LCS

Investigation: MZ, AK, UR, LP

Visualization: $\mathrm{MZ}, \mathrm{AK}, \mathrm{NC}$

Supervision: NC, LCS 
Writing - original draft: $\mathrm{MZ}, \mathrm{AK}, \mathrm{NC}$

Writing- review \& editing: $M Z, A K, N C$

\section{Competing interests}

Authors declare that they have no competing interests.

\section{Data and materials availability}

All data are available in the main text, the supplementary materials as well as in Mendeley Data:

DOI: $10.17632 / c 7 r b x b 86 k 2.1$

\section{References}

1. Tay, M. Z., Poh, C. M., Rénia, L., MacAry, P. A. \& Ng, L. F. P. The trinity of COVID-19: Immunity, inflammation and intervention. Nature Reviews. Immunology 20, 363-374 (2020).

2. Gordon, D. E. et al. A SARS-CoV-2 protein interaction map reveals targets for drug repurposing. Nature 583, 459-468 (2020).

3. Schmidt, N. et al. The SARS-CoV-2 RNA-protein interactome in infected human cells. Nature Microbiology (2020).

4. Atkins, J. F. \& Gesteland, R. F. Recoding: Expansion of decoding rules enriches gene expression. vol. 24 (Springer New York, 2010).

5. Caliskan, N., Peske, F. \& Rodnina, M. V. Changed in translation: mRNA recoding by -1 programmed ribosomal frameshifting. Trends in Biochemical Sciences 40, 265-274 (2015).

6. Caliskan, N., Katunin, V. I., Belardinelli, R., Peske, F. \& Rodnina, M. V. Programmed -1 frameshifting by kinetic partitioning during impeded translocation. Cel/ 157, 1619-1631 (2014).

7. Neupane, K. et al. Anti-frameshifting ligand active against SARS coronavirus-2 is resistant to natural mutations of the frameshift-stimulatory pseudoknot. Journal of Molecular Biology 432, 5843-5847 (2020).

8. Baranov, P. V. et al. Programmed ribosomal frameshifting in decoding the SARS-CoV genome. Virology 332, 498-510 (2005).

9. Brierley, I., Digard, P. \& Inglis, S. C. Characterization of an efficient coronavirus ribosomal frameshifting signal: Requirement for an RNA pseudoknot. Cell 57, 537-547 (1989).

10. Chen, J. et al. Dynamic pathways of -1 translational frameshifting. Nature 512, 328-332 (2014).

11. Matsumoto, S., Caliskan, N., Rodnina, M. V., Murata, A. \& Nakatani, K. Small synthetic moleculestabilized RNA pseudoknot as an activator for -1 ribosomal frameshifting. Nucleic Acids Research 46, 8079-8089 (2018). 
12. Napthine, S. et al. Protein-directed ribosomal frameshifting temporally regulates gene expression. Nature Communications 8, 15582 (2017).

13. Belew, A. T. et al. Ribosomal frameshifting in the CCR5 mRNA is regulated by miRNAs and the NMD pathway. Nature 512, 265-269 (2014).

14. Napthine, S. et al. A novel role for poly(C) binding proteins in programmed ribosomal frameshifting. Nucleic Acids Research 44, 5491-5503 (2016).

15. Wang, X. et al. Regulation of HIV-1 gag-pol expression by shiftless, an inhibitor of programmed -1 ribosomal frameshifting. Cell 176, 625-635.e14 (2019).

16. Kobayashi, Y., Zhuang, J., Peltz, S. \& Dougherty, J. Identification of a cellular factor that modulates HIV-1 programmed ribosomal frameshifting. The Journal of Biological Chemistry 285, 19776-19784 (2010).

17. Butter, F., Scheibe, M., Mörl, M. \& Mann, M. Unbiased RNA-protein interaction screen by quantitative proteomics. Proceedings of the National Academy of Sciences of the United States of America 106, 10626-10631 (2009).

18. Harcourt, J. L., Caidi, H., Anderson, L. J. \& Haynes, L. M. Evaluation of the calu-3 cell line as a model of in vitro respiratory syncytial virus infection. Journal of Virological Methods 174, 144-149 (2011).

19. Castello, A. et al. Insights into RNA biology from an atlas of mammalian mRNA-binding proteins. Cell 149, 1393-1406 (2012).

20. Lee, S. et al. The SARS-CoV-2 RNA interactome. Molecular Cell (2021).

21. Kamel, W. et al. Global analysis of protein-RNA interactions in SARS-CoV-2 infected cells reveals key regulators of infection. BioRxiv (2020).

22. Flynn, R. A. et al. Discovery and functional interrogation of SARS-CoV-2 RNA-host protein interactions. Cell (2021).

23. Geuens, T., Bouhy, D. \& Timmerman, V. The hnRNP family: Insights into their role in health and disease. Human Genetics 135, 851-867 (2016).

24. Wu, J. et al. Cryo-EM structure of the human ribonuclease p holoenzyme. Cell 175, 1393-1404.e11 (2018).

25. Bick, M. J. et al. Expression of the zinc-finger antiviral protein inhibits alphavirus replication. Journal of Virology 77, 11555-11562 (2003).

26. Eswarappa, S. M. et al. Programmed translational readthrough generates antiangiogenic VEGF-ax. Cell 157, 1605-1618 (2014).

27. Friedrich, S. et al. The host factor AUF1 p45 supports flavivirus propagation by triggering the RNA switch required for viral genome cyclization. Journal of Virology 92, (2018).

28. Ray, P. S. \& Das, S. La autoantigen is required for the internal ribosome entry site-mediated translation of coxsackievirus b3 RNA. Nucleic Acids Research 30, 4500-4508 (2002).

29. Weinlich, S. et al. IGF2BP1 enhances HCV IRES-mediated translation initiation via the $3^{\prime} U T R$. RNA (New York) 15, 1528-1542 (2009). 
30. Zhang, J. et al. hnRNPs and ELAVL1 cooperate with UORFs to inhibit protein translation. Nucleic Acids Research (2016).

31. Sauer, M. et al. DHX36 prevents the accumulation of translationally inactive mRNAs with g4structures in untranslated regions. Nature Communications 10, 2421 (2019).

32. Yoo, J.-S. et al. DHX36 enhances RIG-i signaling by facilitating PKR-mediated antiviral stress granule formation. PLoS Pathogens 10, e1004012 (2014).

33. Schwerk, J. et al. RNA-binding protein isoforms ZAP-s and ZAP-I have distinct antiviral and immune resolution functions. Nature Immunology 20, 1610-1620 (2019).

34. Kwak, J. E., Wang, L., Ballantyne, S., Kimble, J. \& Wickens, M. Mammalian GLD-2 homologs are poly (A) polymerases. Proceedings of the National Academy of Sciences of the United States of America 101, 4407-4412 (2004).

35. Pietras, Z. et al. Dedicated surveillance mechanism controls g-quadruplex forming non-coding RNAs in human mitochondria. Nature Communications 9, 2558 (2018).

36. Liang, X. et al. Structural snapshots of human pre-60S ribosomal particles before and after nuclear export. Nature Communications 11, 3542 (2020).

37. Plant, E. P. et al. A three-stemmed mRNA pseudoknot in the SARS coronavirus frameshift signal. PLoS Biology 3, e172 (2005).

38. Syed Lal Badshah, Ullah, A. \& Syed, S. The role of zinc-finger antiviral proteins in immunity against viruses. Molecular Genetics, Microbiology and Virology 35, 78-84 (2020).

39. Li, M. M. H. et al. TRIM25 enhances the antiviral action of zinc-finger antiviral protein (ZAP). PLoS Pathogens 13, e1006145 (2017).

40. Ficarelli, M. et al. KHNYN is essential for the zinc finger antiviral protein (ZAP) to restrict HIV-1 containing clustered CpG dinucleotides. eLife 8, (2019).

41. Meagher, J. L. et al. Structure of the zinc-finger antiviral protein in complex with RNA reveals a mechanism for selective targeting of CG-rich viral sequences. Proceedings of the National Academy of Sciences of the United States of America 116, 24303-24309 (2019).

42. Peng, C. et al. Zinc-finger antiviral protein (ZAP) is a restriction factor for replication of modified vaccinia virus ankara (MVA) in human cells. PLoS Pathogens 16, e1008845 (2020).

43. Manktelow, E., Shigemoto, K. \& Brierley, I. Characterization of the frameshift signal of edr, a mammalian example of programmed -1 ribosomal frameshifting. Nucleic Acids Research 33, 15531563 (2005).

44. Nchioua, R. et al. SARS-CoV-2 is restricted by zinc finger antiviral protein despite preadaptation to the low-CpG environment in humans. mBio 11, (2020).

45. Plant, E. P., Rakauskaite, R., Taylor, D. R. \& Dinman, J. D. Achieving a golden mean: Mechanisms by which coronaviruses ensure synthesis of the correct stoichiometric ratios of viral proteins. Journal of Virology 84, 4330-4340 (2010). 
46. Kim, Y. G., Maas, S., Wang, S. C. \& Rich, A. Mutational study reveals that tertiary interactions are conserved in ribosomal frameshifting pseudoknots of two luteoviruses. RNA (New York) 6, 11571165 (2000).

47. Omar, S. I. et al. Modeling the structure of the frameshift-stimulatory pseudoknot in SARS-CoV-2 reveals multiple possible conformers. PLoS Computational Biology 17, e1008603 (2021).

48. Takata, M. A. et al. CG dinucleotide suppression enables antiviral defence targeting non-self RNA. Nature 550, 124-127 (2017).

49. Bhatt, P. R. et al. Structural basis of ribosomal frameshifting during translation of the SARS-CoV-2 RNA genome. BioRxiv (2020).

50. Zhang, K. et al. Cryo-electron microscopy and exploratory antisense targeting of the 28-kDa frameshift stimulation element from the SARS-CoV-2 RNA genome. BioRxiv (2020).

51. Neupane, K. et al. Structural dynamics of the SARS-CoV-2 frameshift-stimulatory pseudoknot reveal topologically distinct conformers. BioRxiv (2020).

52. Ritchie, D. B., Soong, J., Sikkema, W. K. A. \& Woodside, M. T. Anti-frameshifting ligand reduces the conformational plasticity of the SARS virus pseudoknot. Journal of the American Chemical Society 136, 2196-2199 (2014).

53. Hill, C. H. et al. Structural studies of cardiovirus 2A protein reveal the molecular basis for RNA recognition and translational control. BioRxiv(2020).

54. Lapointe, C. P. et al. Dynamic competition between SARS-CoV-2 NSP1 and mRNA on the human ribosome inhibits translation initiation. Proceedings of the National Academy of Sciences of the United States of America 118, (2021).

55. Zhu, Y., Wang, X., Goff, S. P. \& Gao, G. Translational repression precedes and is required for ZAPmediated mRNA decay. The EMBO Journal 31, 4236-4246 (2012).

56. Rajsbaum, R., García-Sastre, A. \& Versteeg, G. A. TRIMmunity: The roles of the TRIM e3-ubiquitin ligase family in innate antiviral immunity. Journal of Molecular Biology 426, 1265-1284 (2014).

57. Richter, N. J., Rogers, G. W., Hensold, J. O. \& Merrick, W. C. Further biochemical and kinetic characterization of human eukaryotic initiation factor $4 \mathrm{H}$. The Journal of Biological Chemistry 274, 35415-35424 (1999).

58. Philippe, L., Vasseur, J.-J., Debart, F. \& Thoreen, C. C. La-related protein 1 (LARP1) repression of TOP mRNA translation is mediated through its cap-binding domain and controlled by an adjacent regulatory region. Nucleic Acids Research 46, 1457-1469 (2018).

59. McCauley, M. J., Rouzina, I., Li, J., Núñez, M. E. \& Williams, M. C. Significant differences in RNA structure destabilization by HIV-1 GagDp6 and NCp7 proteins. Viruses 12, (2020).

60. Choi, J., O'Loughlin, S., Atkins, J. F. \& Puglisi, J. D. The energy landscape of -1 ribosomal frameshifting. Science Advances 6, eaax6969 (2020).

61. Turakhiya, A. et al. ZFAND1 recruits p97 and the 26S proteasome to promote the clearance of arsenite-induced stress granules. Molecular Cell 70, 906-919.e7 (2018). 
62. Mi, H., Muruganujan, A. \& Thomas, P. D. PANTHER in 2013: Modeling the evolution of gene function, and other gene attributes, in the context of phylogenetic trees. Nucleic Acids Research 41, D377-86 (2013).

63. DeCaprio, J. \& Kohl, T. O. Immunoprecipitation. Cold Spring Harbor Protocols 2017, pdb.prot098640 (2017).

64. Pan, D. et al. CDK-regulated dimerization of M18BP1 on a mis18 hexamer is necessary for CENP-a loading. eLife 6, (2017).

65. Loughran, G., Howard, M. T., Firth, A. E. \& Atkins, J. F. Avoidance of reporter assay distortions from fused dual reporters. RNA (New York) 23, 1285-1289 (2017).

66. Arai, R., Ueda, H., Kitayama, A., Kamiya, N. \& Nagamune, T. Design of the linkers which effectively separate domains of a bifunctional fusion protein. Protein Engineering 14, 529- 532 (2001).

67. Meyer, S. et al. Multi-host expression system for recombinant production of challenging proteins. Plos One 8, e68674 (2013).

68. Gibson, D. G. et al. Enzymatic assembly of DNA molecules up to several hundred kilobases. Nature Methods 6, 343-345 (2009).

69. Andreou, A. I. \& Nakayama, N. Mobius assembly: A versatile golden-gate framework towards universal DNA assembly. Plos One 13, e0189892 (2018).

70. Götzke, H. et al. The ALFA-tag is a highly versatile tool for nanobody-based bioscience applications. Nature Communications 10, 4403 (2019).

71. Brennan, T. V., Lin, L., Huang, X. \& Yang, Y. Generation of luciferase-expressing tumor cell lines. Bioprotocol 8, (2018).

72. Stewart, S. A. et al. Lentivirus-delivered stable gene silencing by RNAi in primary cells. RNA (New York) 9, 493-501 (2003).

73. Grentzmann, G., Ingram, J. A., Kelly, P. J., Gesteland, R. F. \& Atkins, J. F. A dual-luciferase reporter system for studying recoding signals. RNA (New York) 4, 479-486 (1998).

74. Schindelin, J. et al. Fiji: An open-source platform for biological-image analysis. Nature Methods 9 , 676-682 (2012).

75. Kingston, R. E., Chomczynski, P. \& Sacchi, N. Guanidine methods for total RNA preparation. Current Protocols in Molecular Biology Chapter 4, Unit4.2 (2001).

\section{Figures}


A

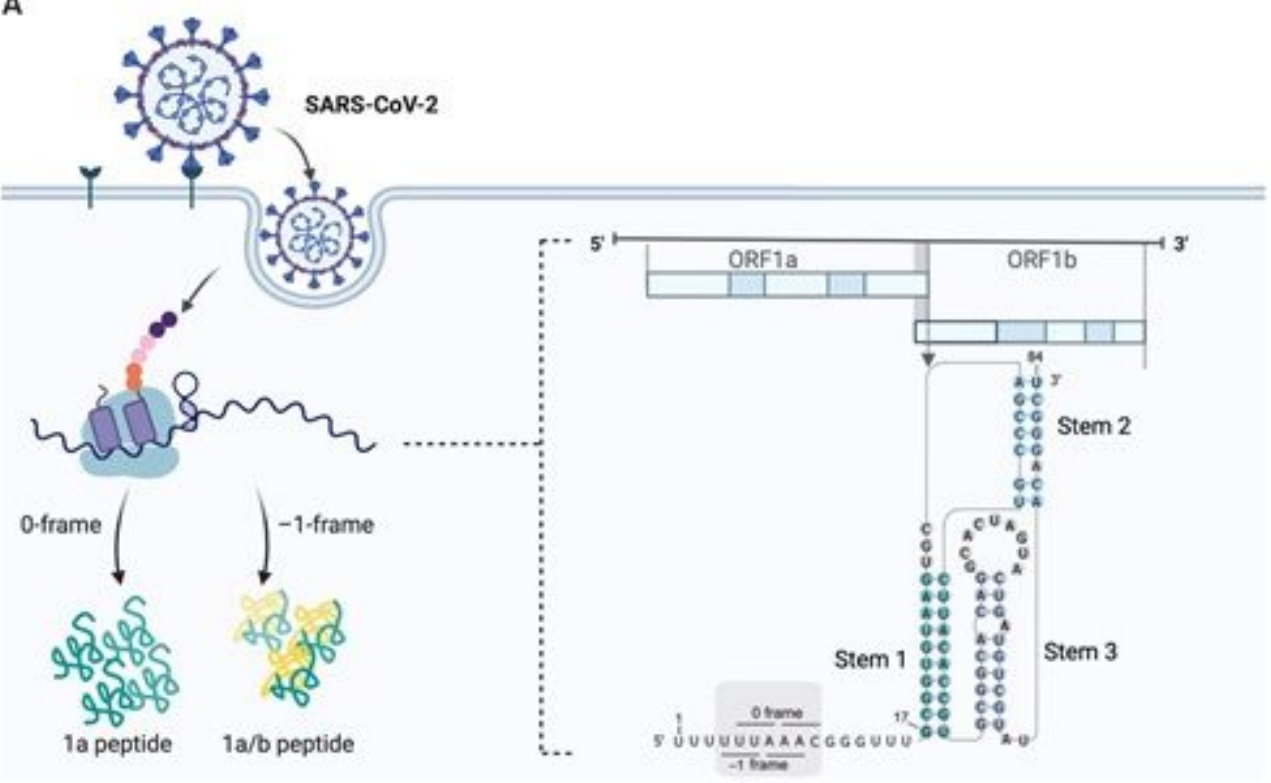

B Infected/uninfected

RNA-Antisense Purification cell lysate

Proteomic Analysis

LC-MS/MS

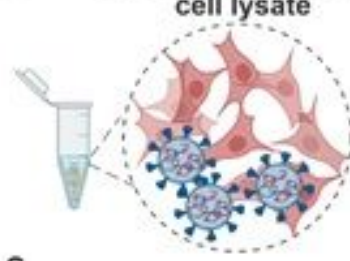

C

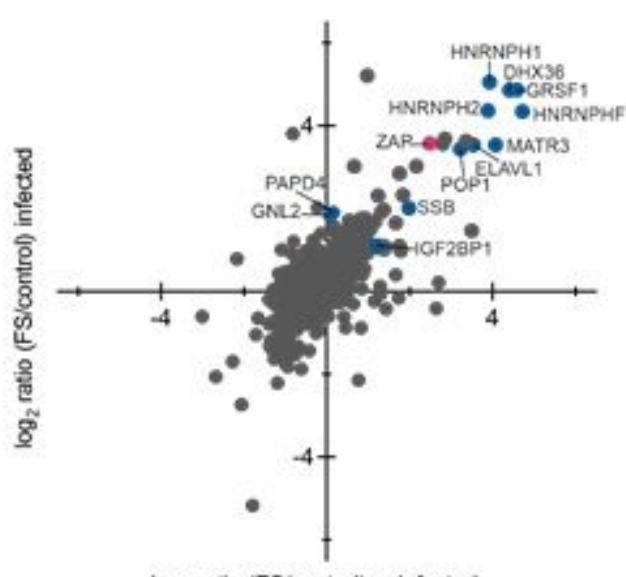

$\log _{2}$ ratio (FS/control) uninfected

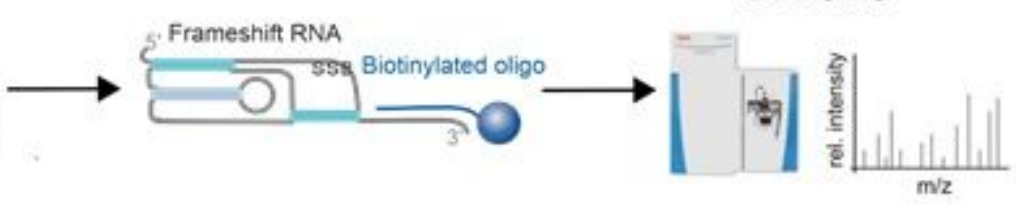

D

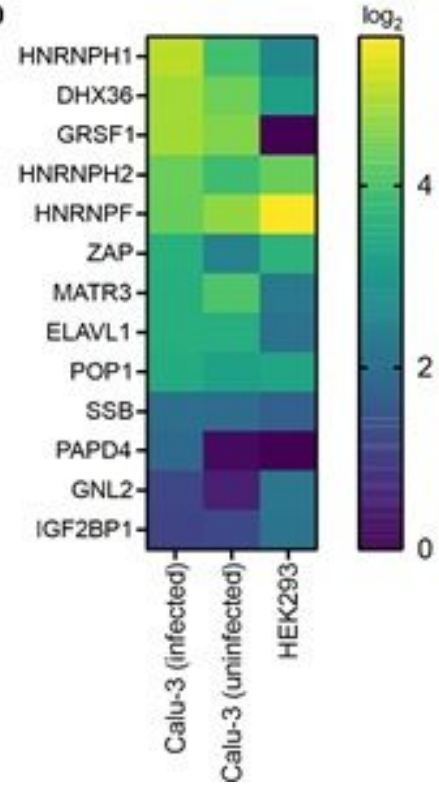

\section{Figure 1}

In vitro RNA-antisense purification-based discovery of protein interactors of the SARS-CoV-2 -1PRF element. (A) Schematic representation of the relevant genomic segment of SARS-CoV-2 as well as the location of the -1PRF element. (B) Schematic of in vitro interactome capture of protein interactors of the SARS-CoV-2 -1PRF element. In vitro synthesized RNA fragments corresponding to nucleotides 1345613570 of the SARS-CoV-2 genome were incubated with lysates of naïve HEK293 cells as well as SARSCoV-2-infected and uninfected Calu-3 cells. The -1PRF RNA was captured by a biotinylated antisense DNA oligo and isolated proteins were subjected to LC-MS/MS. (C) Representative scatter plot of log2- 
ratios comparing proteins captured in uninfected vs. SARS-CoV-2-infected Calu-3 cells. Core interactors common between uninfected and SARS-CoV-2-infected Calu-3 cells as well as uninfected HEK293 cells are highlighted in blue, ZAP is highlighted in pink. (D) Heat map representing the respective enrichment (log2) of core interactors. See also Supplementary Fig. 1D and Supplementary Table 1.

A

Control construct (CC)

CMV

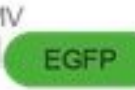

-1 Stop

EGFP

Frameshift construct (-1FS)

CMV

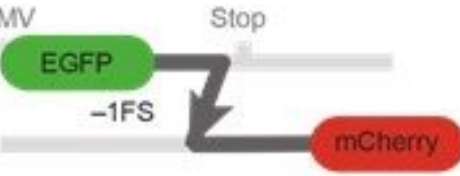

0-frame

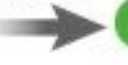

EGFP

EGFP

-1-frame

Trans-factor construct

CMV

\section{ECFP}

\section{Gol}

C

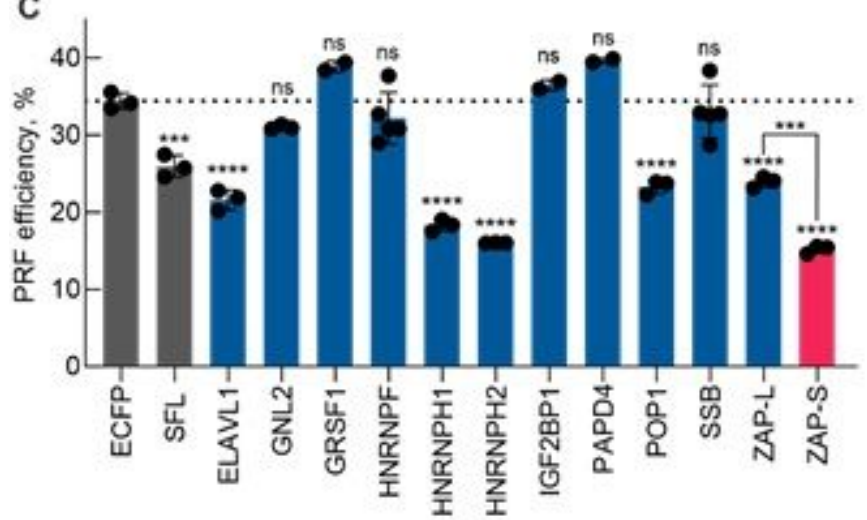

E

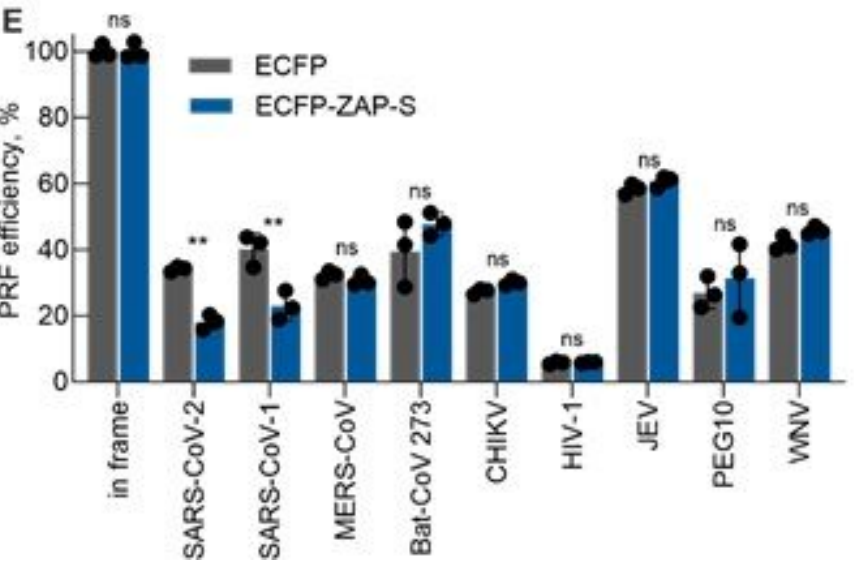

B
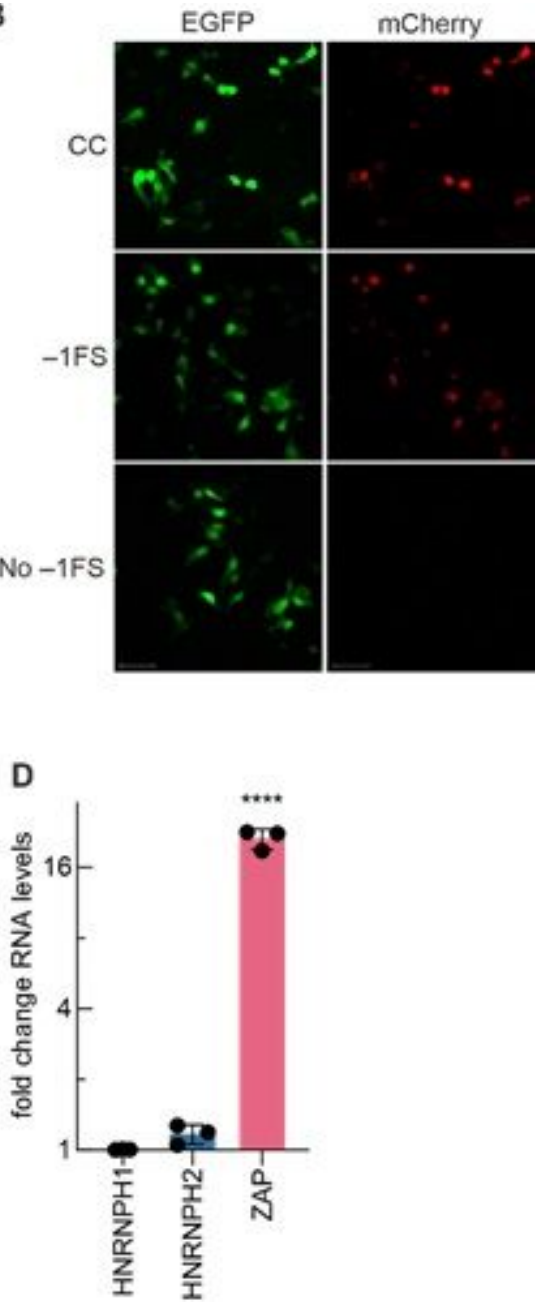

F

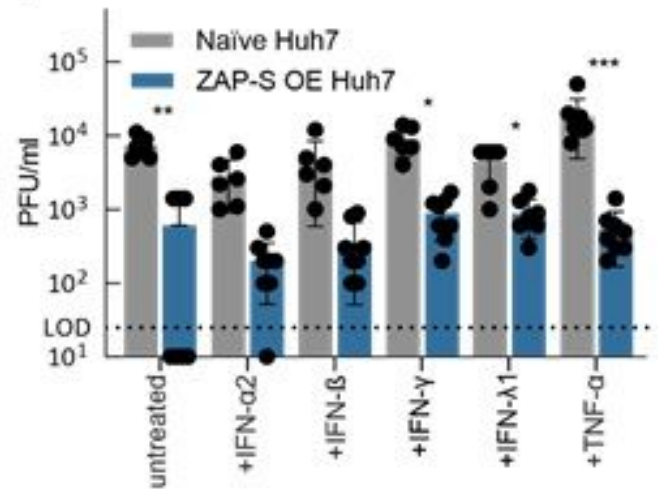

Figure 2

A functional screen of SARS-CoV-2 -1PRF element interactors. (A) Schematic representation of the dualfluorescence frameshift reporter construct. The control construct consists of EGFP in-frame with mCherry separated by a self-cleaving 2A peptide resulting in equimolar production of EGFP and mCherry. In the 
frameshift construct below, EGFP and mCherry are not only separated by a self-cleaving 2A peptide but also by a stop codon in-frame with EGFP. As a result, 0-frame translation would produce only EGFP, whereas -1PRF would produce both EGFP and mCherry. The ratio of mCherry to GFP fluorescence is used to quantify the FE. The trans-factor construct is an N-terminal fusion of ECFP with the protein of interest to be analyzed. The control construct consists of ECFP alone. (B) Confocal microscopy images of cells transfected with EGFP-mCherry control (CC), -1FS, and no FS (no -1PRF site included after EGFP and mCherry in-frame with EGFP) constructs. The size bar represents $50 \mu \mathrm{m}$. (C) Comparison of relative FE of cells overexpressing trans-factors as ECFP fusion proteins. Data points represent the mean $\pm \mathrm{s}$.d. (n $=3$ independent experiments). $P$ values were calculated using an ordinary unpaired one-sided ANOVA comparing every condition to the ECFP control. ZAP-L and ZAP-S were separately compared to each other. ${ }^{*} P<0.01-* \star P<0.001-* \star \star P<0.0001-* \star \star \star P<0.00001$. (D) Expression profiles of selected genes in Calu-3 infected cells with SARS-CoV-2 at 72 hours. RNA levels were quantified by qRT-PCR and normalized to the respective RNA abundance in uninfected cells (shown as 2- $\triangle \triangle C T$ ). Data points represent the mean \pm s.d. ( $n=3$ independent experiments). $P$ values were calculated using an ordinary unpaired one-sided ANOVA comparing delta CT values of the respective RNA in uninfected and infected

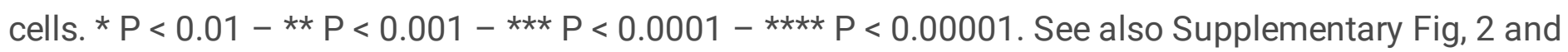
Supplementary Table 2. (E) In vivo dual-fluorescence of additional -1PRF RNAs in HEK293 cells in the presence and absence of ZAP-S. SARS-CoV-1 - Severe acute respiratory syndrome-related Coronavirus 1 , MERS-CoV - Middle East respiratory syndrome-related Coronavirus, Bat-CoV-273 - Bat Coronavirus 273, CHIKV - Chikungunya Virus, HIV-1 - Human Immunodeficiency Virus 1, JEV - Japanese Encephalitis Virus, WNV - West Nile Virus. Data points represent the mean \pm s.d. $(n=3$ independent experiments). $P$ values were calculated using an ordinary unpaired one-sided ANOVA comparing every condition to the ECFP control. * $P<0.01-* * P<0.001$. (F) Virus titers in the supernatant of infected naïve Huh7 or ZAP-S overexpressing Huh7 cells (ZAP-S OE) at 24 hours post infection. Treatment with IFN- $\mathbb{(}(500 \mathrm{U} / \mathrm{ml})$, IFN- $\beta$ $(500 \mathrm{U} / \mathrm{ml})$, or IFN- $₫ 1(5 \mathrm{ng} / \mathrm{ml})$ was done one hour before infection. Boxes show mean values $\pm \mathrm{s} . \mathrm{d}$. $(\mathrm{n}=$ 4). The dotted line represents the limit of detection (LOD). ND: not detected. 


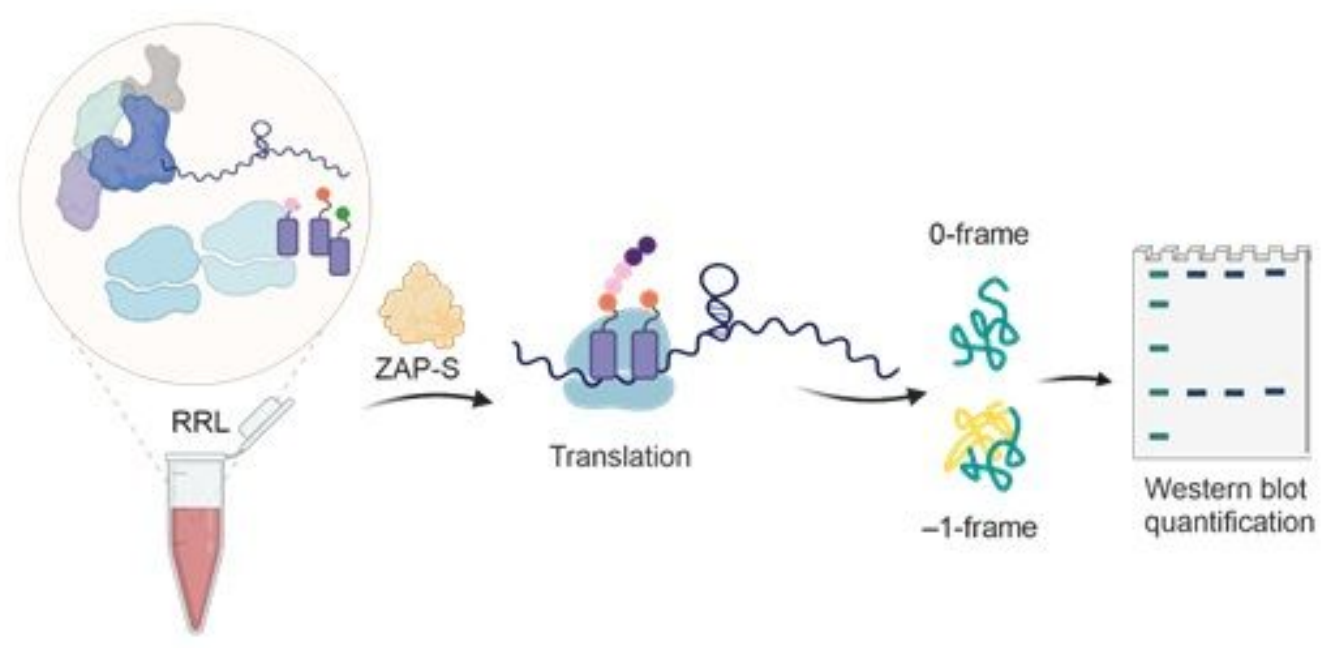

B
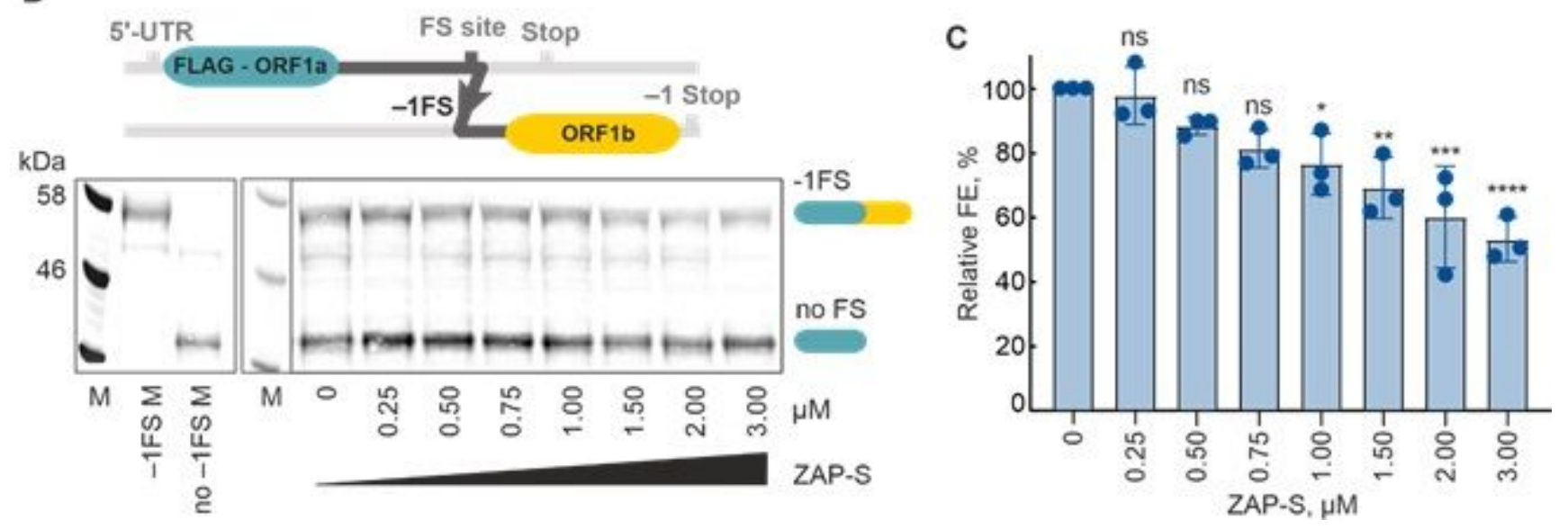

Figure 3

Effect of zinc-finger antiviral protein (ZAP) on $1 \mathrm{a} / 1 \mathrm{~b}-1$ frameshifting in vitro. (A) The strategy of the in vitro translation assay using rabbit reticulocyte lysate (RRL). (B) Schematics of the N-terminal FLAGtagged frameshifting reporter consisting of the nucleotides 12686-14190 ( 1.5kb) of the SARS-CoV-2 genome. RNAs were translated in RRL in the presence of increasing concentrations of ZAP-S ranging from 0 to $3 \mu \mathrm{M}$. FLAG-tagged peptides generated by ribosomes that do not frameshift (no $-1 \mathrm{PRF}$ ) or that enter the -1 reading frame (-1PRF) were identified via western blotting using anti-DDDDK antibody. FE was calculated as previously described 11, by the formula: Intensity (-1-frame)/ (Intensity (-1-frame) + Intensity (0-frame)). Size markers - M (Marker), -1PRF M (-1 frame marker), and no -1PRF M (0-frame marker). (C) Changes in FE observed in the presence of ZAP-S from (B) (normalized to $0 \mu M$ ZAP as shown in $\mathrm{B}$ ). $\mathrm{P}$ values were calculated using an ordinary unpaired one-sided ANOVA comparing every concentration to the no ZAP control. * $P<0.01-* \star P<0.001-* \star \star P<0.0001-* \star \star \star P<0.00001$. See also Supplementary Fig. 2 and Supplementary Table 6. 

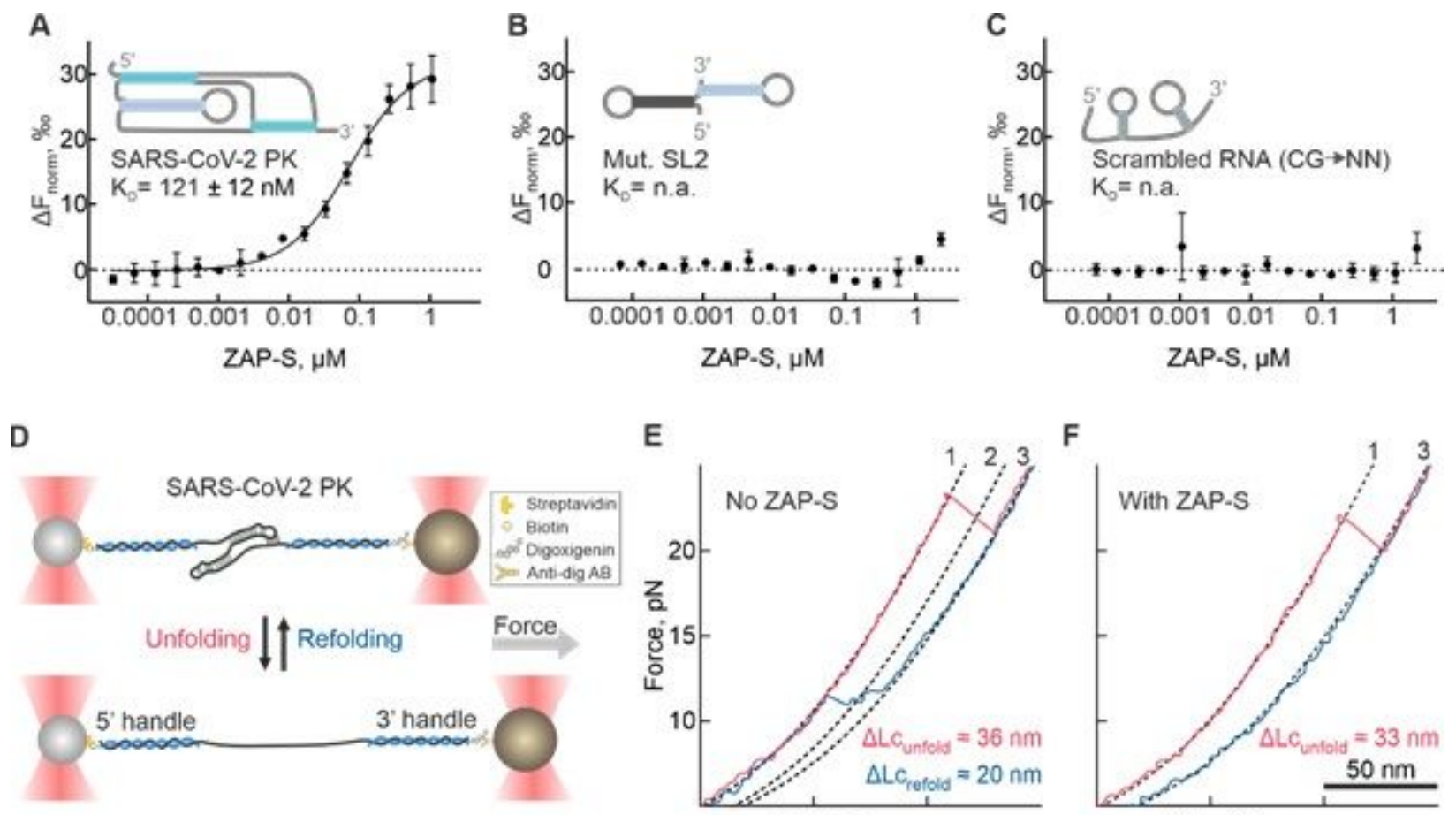

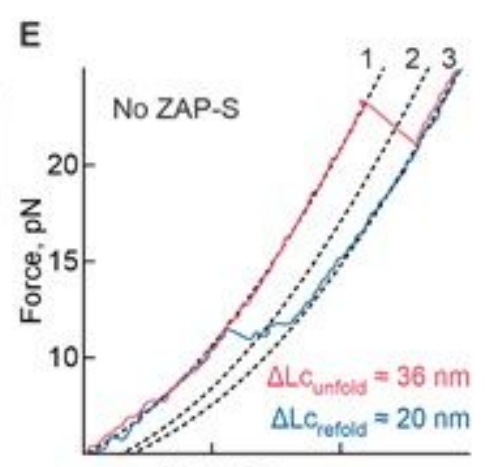

Rel, distance, $\mathrm{nm}$

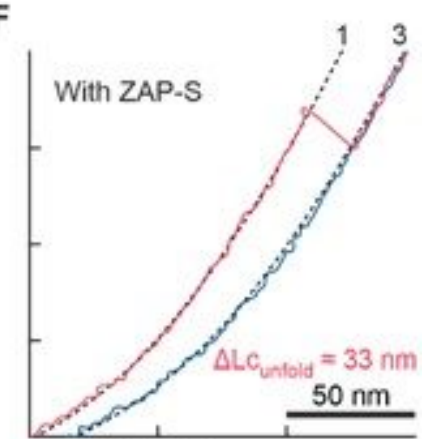

Rel. distance, $\mathrm{nm}$

\section{Figure 4}

In vitro characterization of ZAP-S interaction with SARS-CoV-2 -1 PRF RNA. (A-C) Microscale thermophoresis assay to monitor ZAP-S binding to (A) SARS-CoV-2 pseudoknot, (B) SL2 mutant, (C) CGdepleted scrambled (random structure). Unlabeled ZAP-S (40 pM to $2 \mu \mathrm{M})$ was titrated against 3' pCp-Cy5 labeled RNA ( $5 \mathrm{nM})$ and thermophoresis was recorded at $25^{\circ} \mathrm{C}$ with $5 \%$ LED intensity and medium MST power. Change in fluorescence $(\Delta$ Fnorm) was measured at MST on-time of $5 \mathrm{~s}$. Data were analyzed and $\mathrm{KD}$ was determined using standard functions in the MO. Affinity Analysis software. Data represent mean \pm SD of three measurements $(n=3)$. (D) Schematic illustrating optical tweezers experiments. RNA was hybridized to single-stranded DNA handles flanking the SARS-CoV-2 frameshift site, and conjugated to functionalized beads. A focused laser beam was used to exert pulling force from one end of the molecule. The force was gradually increased until the RNA is fully unfolded (bottom). (E) Example unfolding (pink) and refolding (blue) traces of a SARS-CoV-2 RNA sample. (F) Example unfolding (pink) and refolding (blue) traces of the SARS-CoV-2 RNA in presence of $400 \mathrm{nM}$ ZAP-S. The numbered and dashed lines represent WLC-based fits (from left to right) of (1) folded, (2) intermediate, and (3) unfolded states as described in Methods. See also Supplementary Fig. 3 and S4, Supplementary Table 5. 
A

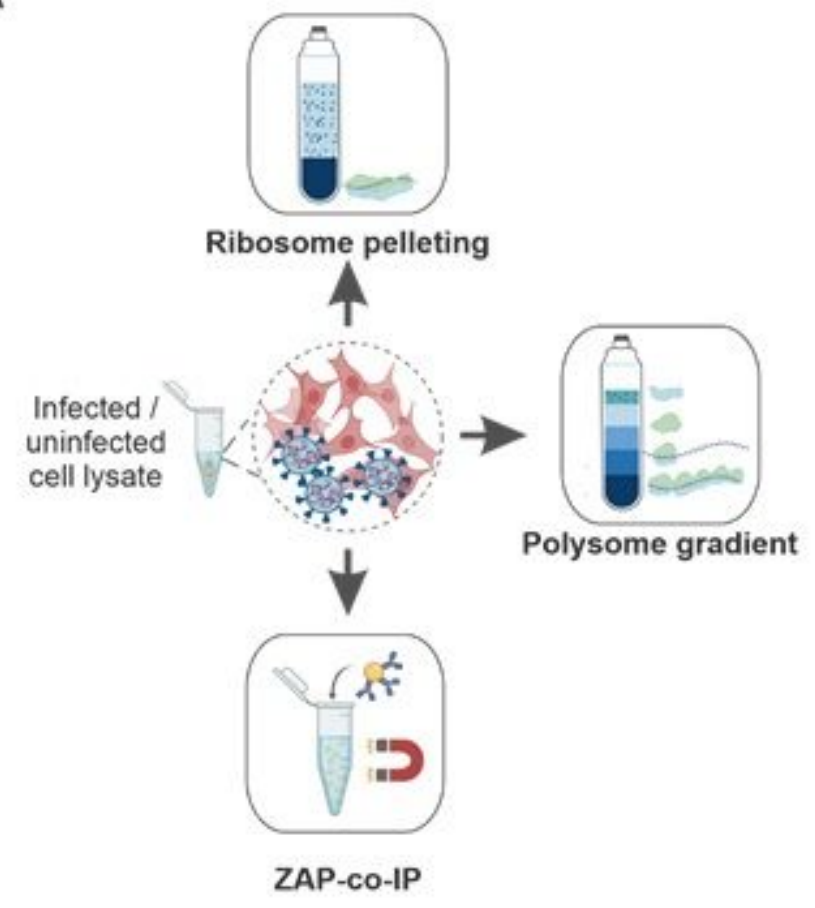

B

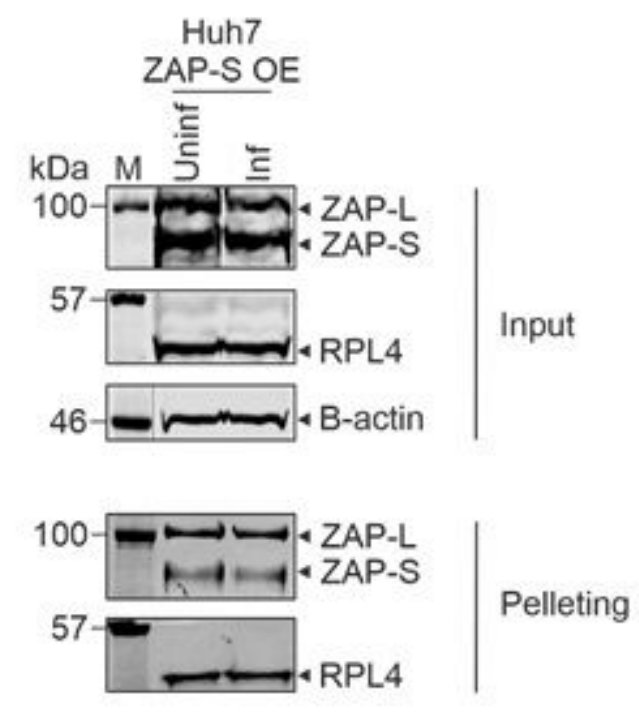

C

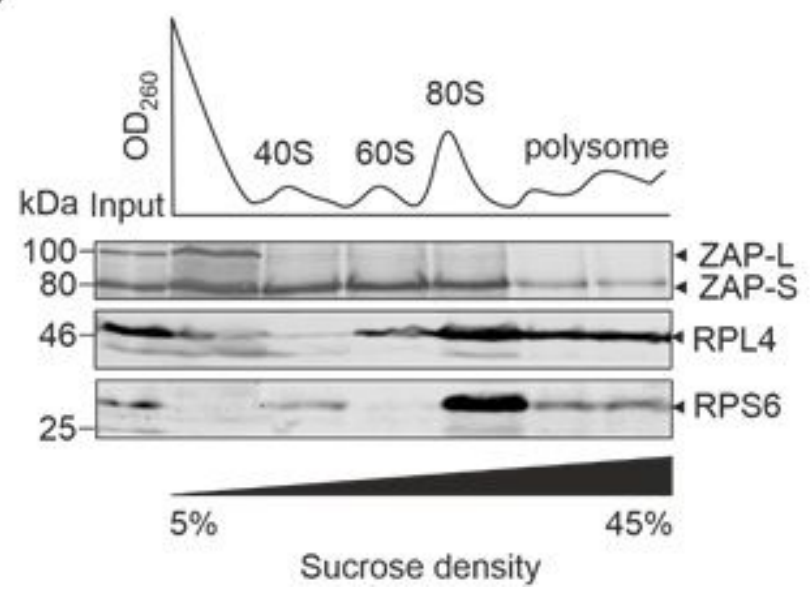

D

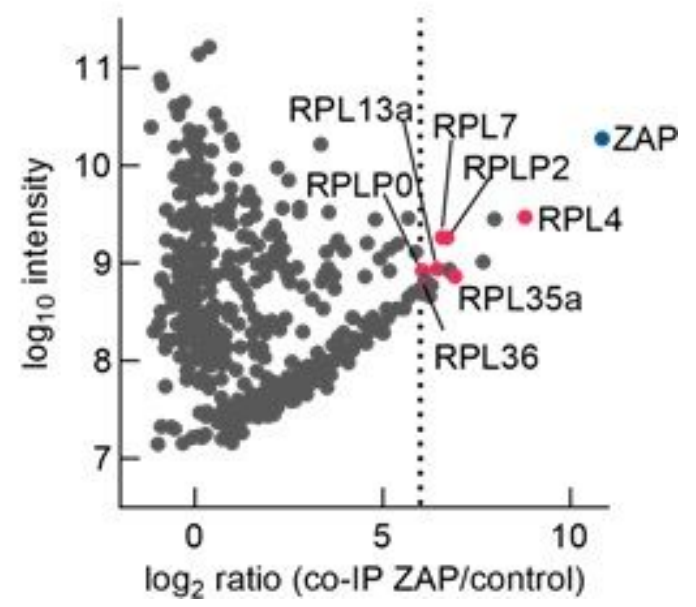

Figure 5

Interactions of ZAP-S with the translation machinery. (A) Schematic of the experimental workflow to analyze ribosome association. (B) Ribosome pelleting of infected cells. Uninfected and SARS-CoV-2 infected $\mathrm{HuH}-7$ cells stably expressing ZAP-S were lysed and loaded onto sucrose cushions. Levels of ribosomal proteins, ZAP, and B-actin in the pellets were analyzed by western blotting using anti-RPL4, anti-RPS6, anti-ZC3HAV1 (ZAP) and anti- beta actin antibodies. (C) Polysome profiling analysis of ZAP. HEK293 cells both naïve and transiently expressing ZAP-S were lysed, subjected to 5-45\% sucrose gradient ultracentrifugation, and subsequently fractionated. Levels of ribosomal proteins, as well as ZAP in each fraction, were analyzed by western blotting using anti-RPL4, anti-RPS6, and anti-ZC3HAV1 (ZAP) antibodies. (D) Ratio vs. intensity plot of a co-immunoprecipitation of ZAP from SARS-CoV-2-infected Calu-3 lysate. Highlighted proteins were enriched by at least 6-fold in comparison to control rabbit lgG. A full list of hits can be found in Supplementary Table 3. 

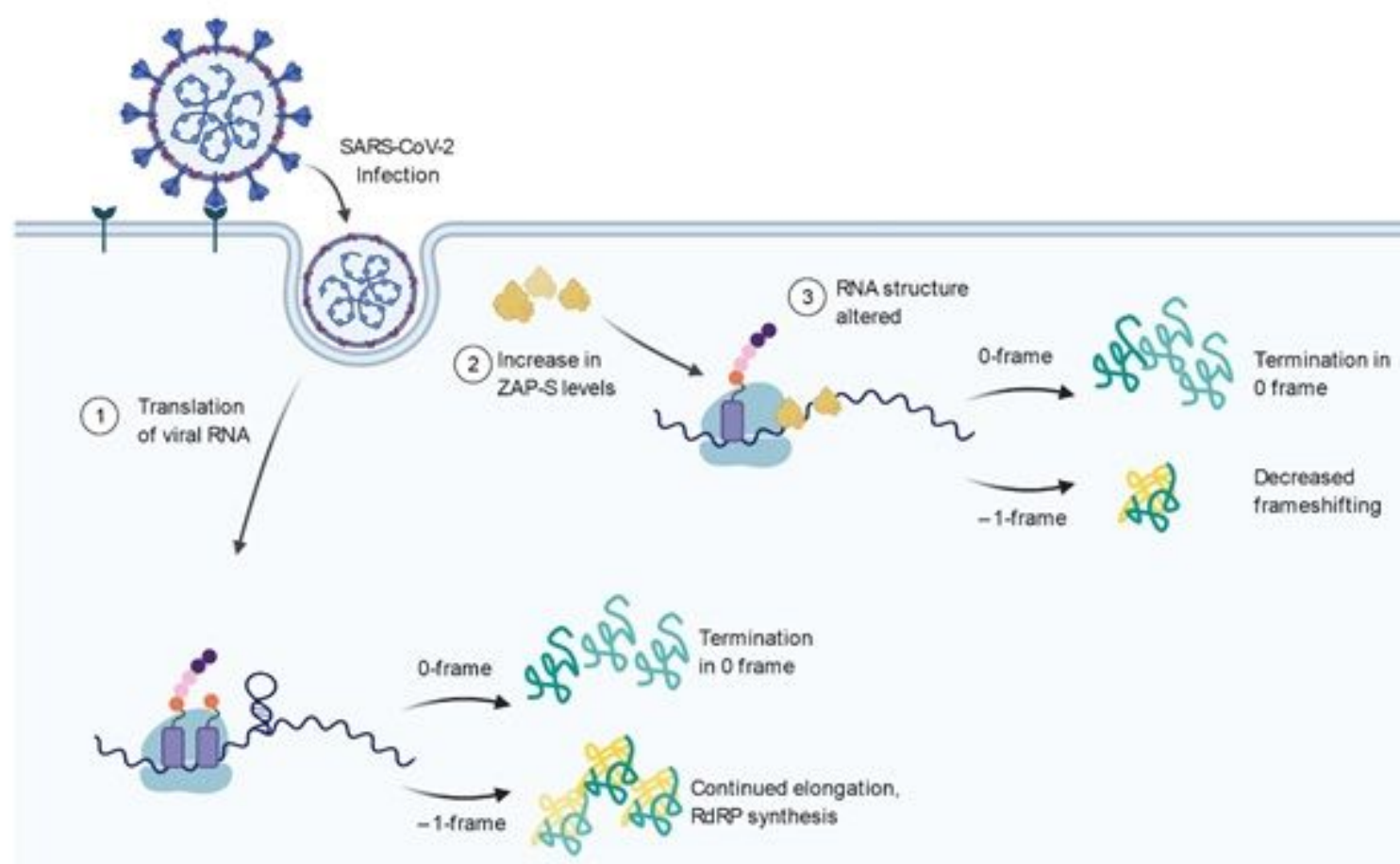

\section{Figure 6}

Model for ZAP-S mediated inhibition of SARS-CoV-2 frameshifting. (1) Upon infection, the viral RNA is translated by the cellular machinery, where $40 \%$ of translation events yield the $1 \mathrm{a} / 1 \mathrm{~b}$ polyprotein through -1PRF. (2) Infection also leads to the induction of antiviral factors including ZAP-S. (3) ZAP-S binding to the frameshift RNA alters the RNA structure and reduces the chance of elongating ribosomes to encounter the stimulatory structure. In absence of this structure, the elongation pause would be too short for codon-anti-codon interactions to be established in the -1 frame. Thus, ZAP-S would allow translation to proceed without any roadblock effect and terminate at the canonical 0-frame UAA stop codon found just downstream of the slippery sequence. This would lead to a decrease in the amounts of the $1 \mathrm{a} / 1 \mathrm{~b}$ polypeptides and thus hamper the production of the RdRP from the -1 frame.

\section{Supplementary Files}

This is a list of supplementary files associated with this preprint. Click to download.

- SupplementaryTable1invitroRNAantisensepurificationMS.xlsx

- SupplementaryTable2Rawflowcytometrydata.xlsx

- SupplementaryTable3ZAPcolPhits.xIsx

- SupplementaryTable4DNAoligonucleotideandplasmidsequences.xlsx 
- supplementarytable5ZAPSOT.docx

- SupplementaryTable6invitrotranslations.xIsx

- SupplementaryFigure15.pdf 